\title{
Explicit Integration of Friedmann's Equation with Nonlinear Equations of State
}

\author{
Shouxin Chen* \\ Institute of Contemporary Mathematics \\ School of Mathematics \\ Henan University \\ Kaifeng, Henan 475004, PR China \\ Gary W. Gibbons ${ }^{\dagger}$ \\ D. A. M. T. P. \\ University of Cambridge \\ Cambridge CB3 0WA, U. K. \\ Yisong Yang \\ Department of Mathematics \\ Polytechnic School, New York University \\ Brooklyn, New York 11201, U. S. A \\ $\&$ \\ NYU-ECNU Institute of Mathematical Sciences \\ New York University - Shanghai \\ 3663 North Zhongshan Road, Shanghai 200062, PR China
}

\begin{abstract}
In this paper we study the integrability of the Friedmann equations, when the equation of state for the perfect-fluid universe is nonlinear, in the light of the Chebyshev theorem. A series of important, yet not previously touched, problems will be worked out which include the generalized Chaplygin gas, two-term energy density, trinomial Friedmann, Born-Infeld, two-fluid models, and Chern-Simons modified
\end{abstract}

${ }^{*}$ Email address: chensx@henu.edu.cn

${ }^{\dagger}$ Email address: gwg1@damtp.cam.ac.uk

†Email address: yisongyang@nyu.edu 
gravity theory models. With the explicit integration, we are able to understand exactly the roles of the physical parameters in various models play in the cosmological evolution which may also offer clues to a profound understanding of the problems in general settings. For example, in the Chaplygin gas universe, a few integrable cases lead us to derive a universal formula for the asymptotic exponential growth rate of the scale factor, of an explicit form, whether the Friedmann equation is integrable or not, which reveals the coupled roles played by various physical sectors and it is seen that, as far as there is a tiny presence of nonlinear matter, conventional linear matter makes contribution to the dark matter, which becomes significant near the phantom divide line. The Friedmann equations also arise in areas of physics not directly related to cosmology. We provide some examples ranging from geometric optics and central orbits to soap films and the shape of glaciated valleys to which our results may be applied.

Keywords: Astrophysical fluid dynamics, cosmology with extra dimensions, alternatives to inflation, initial conditions and eternal universe, cosmological applications of theories with extra dimensions, string theory and cosmology.

PACS numbers: 04.20.Jb, 98.80.Jk

\section{Contents}

1 Introduction

2 Friedmann's Equation and Chebyshev's Theorem 5

2.1 Reduced Temperature . . . . . . . . . . . . . . . . 6

2.2 Conformal Time . . . . . . . . . . . . . . . . . . . 6

2.3 Chebyshev's Theorem for Binomial Integrals . . . . . . . . . . . . . 7

3 Perfect Fluids 7

3.1 Equations of Motion and Thermodynamics . . . . . . . . . . . . 7

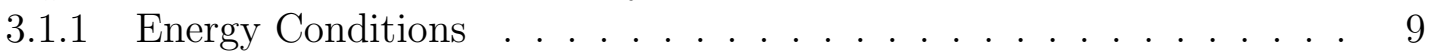

3.1.2 Examples . . . . . . . . . . . . . . . . . . . . . 9

3.2 Dark Energy . . . . . . . . . . . . . . . . . . . . 10

3.3 Stationary Flows . . . . . . . . . . . . . . . . . . . . . . . . . . . . . . . . . . . .

3.4 Irrotational Fluids . . . . . . . . . . . . . . . . . . . . . . . . 11

3.5 Lagrangian Description of Irrotational Perfect Fluids . . . . . . . . . . . . 11

3.6 Application to Friedmann-Lemaître Cosmologies . . . . . . . . . . . . . . . 12

4 The Chaplygin Gas and the Born-Infeld Models 14

4.1 The Chaplygin Gas Model . . . . . . . . . . . . . . . . . . . . . . . . 14

4.2 A Universal Formula for Asymptotic Exponential Growth Rate and Cosmological Consequences . . . . . . . . . . . . . . . 18

4.3 The Born-Infeld Type Fluid Model . . . . . . . . . . . . . . . . . . . . . . 20 
5 Multiple Fluids 23

5.1 A Two-Term Energy Density Model . . . . . . . . . . . . . . . . . . 23

5.2 A Two-Fluid Model . . . . . . . . . . . . . . . . . . . . . . 25

5.3 A Trinomial Friedmann Equation . . . . . . . . . . . . . 28

5.4 Friedmann's Equation in a Chern-Simons Modified Gravity Theory . . . . 30

6 The Reduced Temperature Equation 33

6.1 Governing Equation in Conformal Time . . . . . . . . . . . . . . 33

6.2 Integration of Equation . . . . . . . . . . . . . . . . . 34

7 Friedmann Type Equations in Other Contexts 36

7.1 Refraction in a Horizontally Stratified Medium . . . . . . . . . . 36

7.2 Soap Films and Glaciated Valleys . . . . . . . . . . . . . . . . 38

7.3 Catenary of Equal Strength . . . . . . . . . . . . . . . . . . 40

7.4 The Elastica of Bernoulli and the Capillary Curve . . . . . . . . . . . . . 41

7.5 Central Orbits . . . . . . . . . . . . . . . . . . . . . . 45

7.6 Spherical Symmetric Lenses . . . . . . . . . . . . . . . . . . . 45

8 Conclusion 47

\section{Introduction}

The Einstein equations of general relativity reduce, when the spacetime metric is of the Friedmann-Lemaitre-Robertson-Walker type governing an isotropic and homogeneous universe filled with a perfect fluid characterized by its pressure $P_{m}$ and energy density $\rho_{m}$, to the Friedmann equations: a set of nonlinear ordinary differential equations, determining the law of evolution of the spatial scale factor $a(t)$, in terms of the Hubble "constant", $H=$ $\frac{\dot{a}}{a}$. It is a challenging task, not always possible, to obtain a complete explicit integration in finite terms. In the recent work [1], we demonstrated a link between a theorem of Chebyshev and the explicit integration in both cosmological time $t$ and conformal time $\eta$ of the Friedmann equations in all dimensions and with an arbitrary cosmological constant $\Lambda$. We were able to establish that for spatially flat universes an explicit integration in $t$ may always be carried out, and that, in the non-flat situation and when $\Lambda$ is zero and the ratio $w$ of $P_{m}$ and $\rho_{m}$ in the barotropic equation of state of the perfect-fluid universe is rational, an explicit integration may be carried out if and only if the dimension $n$ of space and $w$ obey some specific relations among an infinite family. Besides, we proved that the situation for an explicit integration in $\eta$ is complementary to that in $t$ so that in the flat-universe case with $\Lambda \neq 0$ an explicit integration in $\eta$ can be carried out if and only if $w$ and $n$ obey similar relations among a well-defined family, and that, when $\Lambda=0$, an explicit integration can always be carried out whether the space is flat, closed, or open. Furthermore, we illustrated with an example that our method is also useful in the study of more realistic cosmological situations when the equation of state is nonlinear. The purpose of our current paper to explore the method further to get exact integrations of 
some other important cases which are not covered in [1] and give a few examples whose integrability is beyond the reach of the Chebyshev theorem but can be obtained by other means. We shall also see how the integration helps understand some general situations when integration is not possible.

When the equation of state of the perfect-fluid universe is nonlinear, the situations include the generalized Chaplygin gas, two-term energy density, the trinomial Friedmann, 1 Born-Infeld, and two-fluid models. Specifically, for the generalized Chaplygin gas model, we work on the flat-universe situation with zero cosmological constant and identify all integrable cases. For the two-term energy density model, we can do the same. In both situations the Chebyshev theorem may be applied directly. In the trinomial Friedmann equation model, we show that the Chebyshev theorem is applicable only in the bottom three-dimensional space case. For the Born-Infeld type fluid model and assuming a flat universe, we show that the Chebyshev theorem works only in the critical coupling situation when the cosmological constant and Newton constant fulfill a specified condition. However, in all non-critical cases, we show that the Friedmann equation allows an integration for any value of the cosmological constant. Moreover, we study a two-fluid model and carry out its integration by the Chebyshev theorem for a closed universe situation. Its interest is that in the original setting the integrand is not of a binomial type but it may be recast and decomposed into a binomial form so that the Chebyshev theorem is applicable. We will also conduct a study of the two-fluid model in terms of the reduced temperature, given as the inverse of the scale factor. In this situation the Friedmann equation in conformal time does not allow an application of the Chebyshev theorem because it cannot be reduced into a binomial form but may still be integrated explicitly when the cosmological constant vanishes and universe is either closed or open (the flat case is trivial). The necessity of introducing several layers of intermediate variables in the process of integration often makes it complicated and cumbersome to express the final dependence of the scale factor on either cosmic or conformal time transparently, although such a task is always manageable. As illustrations, we will work out concrete examples for the models which are integrated in terms of cosmic time. These include the Chaplygin gas, Born-Infeld, two-term energy, two-fluid, trinomial Friedmann equation, and Chern-Simons modified gravity theory models. Although these examples are of different technical features, the common lesson gained is that integration allows us to obtain both qualitatively and quantitatively accurate knowledge about the solutions, especially regarding the roles played by various physical parameters. It should be emphasized that the study on integrable cases may often shed profound insight into nonintegrable cases as well. Here we carry out such a study for the important Chaplygin gas model. We will see that some integrable examples lead us to uncover a universal asymptotic exponential growth rate formula for the expansion of the scale factor regardless whether the equation is integrable. The importance of this formula is that it reveals explicitly the roles played by various coupling parameters and indicates that, no matter how small the nonlinear Chaplygin component is, the conventional linear component always join force to contribute to the exponential

\footnotetext{
${ }^{1}$ That is, the energy density considered as a function of the scale factor contains three terms, each a power of the scale factor.
} 
rate, or dark matter, even though the cosmological constant is absent. Moreover, this universal formula indicates that, near the so-called phantom divide line, the linear component contributes to dark matter significantly. Finally, we will also present a collection of examples of the Friedmann type equations which may be integrated in view of the Chebyshev theorem but fall out of the subject of relativistic cosmology.

The plan of the paper is as follows. In Section 2 we introduce the Friedmann equation and the relevance of the Chebyshev theorem to its integration. The equation is usually expressed in terms of the scale factor $a(t)$, but as, we point out, is sometimes advantageous to express the scale factor as a function of cosmic time or to express the so-called reduced temperature or inverse of the scale factor in terms of conformal time. In Section 3 we review some material about perfect fluids in cosmology. In particular we show how starting from an equation of state, we may always express the energy density as a function of the scale factor and conversely to obtain the equation of state, given the energy density as a function of the scale factor. We also review the Lagrangian approach to perfect fluids and its relation to $k$-essence and similar models of current interest. In Section 4 we consider in detail the Chaplygin gas and the Born-Infeld models. Section 5 is concerned with various fluids with multiple components of the type described above. In Section 6 we discuss the integration of the reduced temperature form of the Friedmann equation. Finally, in Section 7, we point out various occurrences of the Friedmann equation in non-cosmological contexts and the relevance of our results to them. In Section 8 we provide a conclusion and indicate possible future directions.

\section{Friedmann's Equation and Chebyshev's Theorem}

As in [1, the universe we consider here is an $(n+1)$-dimensional homogeneous and isotropic Lorentzian spacetime with the metric

$$
\mathrm{d} s^{2}=g_{\mu \nu} \mathrm{d} x^{\mu} \mathrm{d} x^{\nu}=-\mathrm{d} t^{2}+a^{2}(t) g_{i j} \mathrm{~d} x^{i} \mathrm{~d} x^{j}, \quad i, j=1, \ldots, n,
$$

where $t$ is the cosmological (or cosmic) time and $g_{i j}$ is the metric of an $n$-dimensional Riemannian manifold $M$ of constant scalar curvature characterized by an indicator, $k=$ $-1,0,1$, so that $M$ is an $n$-hyperboloid, the flat space $\mathbb{R}^{n}$, or an $n$-sphere, with the respective metric

$$
g_{i j} \mathrm{~d} x^{i} \mathrm{~d} x^{j}=\frac{1}{1-k r^{2}} \mathrm{~d} r^{2}+r^{2} \mathrm{~d} \Omega_{n-1}^{2},
$$

where $r>0$ is the radial variable and $\mathrm{d} \Omega_{n-1}^{2}$ denotes the canonical metric of the unit sphere $S^{n-1}$ in $\mathbb{R}^{n}$. Inserting the metric (2.1)-(2.2) into the Einstein equations

$$
G_{\mu \nu}+\Lambda g_{\mu \nu}=8 \pi G_{n} T_{\mu \nu}
$$

where $G_{\mu \nu}$ is the Einstein tensor, $G_{n}$ the universal gravitational constant in $n$ spatial dimensions, and $\Lambda$ the cosmological constant, the speed of light is set to unity, and $T_{\mu \nu}$ is the energy-momentum tensor of an ideal cosmological fluid given by

$$
T_{\mu}^{\nu}=\operatorname{diag}\left\{-\rho_{m}, P_{m}, \ldots, P_{m}\right\}
$$


with $\rho_{m}$ and $P_{m}$ the $t$-dependent matter energy density and pressure, we arrive after some standard reduction at the Friedmann equation

$$
H^{2}=\frac{16 \pi G_{n}}{n(n-1)} \rho-\frac{k}{a^{2}}
$$

where $H=\frac{\dot{a}}{a}$ is the usual Hubble parameter, $\dot{f}=\frac{\mathrm{d} f}{\mathrm{~d} t}$, and $\rho, P$ are the effective energy density and pressure given by

$$
\rho=\rho_{m}+\frac{\Lambda}{8 \pi G_{n}}, \quad P=P_{m}-\frac{\Lambda}{8 \pi G_{n}},
$$

subject to the energy-conservation law

$$
\dot{\rho}+n(\rho+P) H=\dot{\rho}_{m}+n\left(\rho_{m}+P_{m}\right) H=0 .
$$

\subsection{Reduced Temperature}

It is of interest to consider another variable, called in [37] the "reduced temperature", of the form

$$
\tau=\frac{C_{0}}{a},
$$

where $C_{0}>0$ is a constant, so that at the big bang when $a=0$ we have infinite "temperature" and the expansion of the universe leads to a decay of this "temperature". Strictly speaking $\tau$ is proportional to the redshift $1+z=\frac{a\left(t_{0}\right)}{a(t)}$ and since the wavelength of freely propagating particles redshifts by this factor and if those particles are in thermodynamic equilibrium then $\tau$ will be proportional to their temperature $T$. For the standard case of radiation for which the energy density is proportional to $a^{-(n+1)}$ in $n$ spatial dimensions the distinction between the reduced temperature and the temperature of the fluid may be ignored. However more generally the two are not the same. For example in three spatial dimensions, if $P=(\gamma-1) \rho$ with $\gamma \neq \frac{4}{3}$ a constant we shall see in Subsection 3.1.1 that $\rho \propto T^{\frac{\gamma}{\gamma-1}}$ while $\rho \propto \tau^{3 \gamma}$. Thus only when $\gamma=\frac{4}{3}$, i.e. for radiation, can the two temperatures be identified.

\subsection{Conformal Time}

It is often convenient to introduce conformal time $\eta$ by

$$
\mathrm{d} t=a \mathrm{~d} \eta
$$

We shall adopt the notation $f^{\prime}=\frac{\mathrm{d} f}{\mathrm{~d} \eta}$. Then $f^{\prime}=f \dot{f}$ and (2.5) becomes

$$
\left(a^{\prime}\right)^{2}=\frac{16 \pi G_{n}}{n(n-1)} \rho a^{4}-k a^{2} .
$$




\subsection{Chebyshev's Theorem for Binomial Integrals}

We have seen that integration of (2.5) or (2.10) requires knowledge whether the binomial integral

$$
I=\int x^{p}\left(\alpha+\beta x^{r}\right)^{q} \mathrm{~d} x
$$

where $p, q, r(r \neq 0)$ are rational numbers and $\alpha, \beta$ are nonzero real numbers, may be represented in terms of elementary functions. As seen in [1], the Chebyshev theorem [2, 3] is what is needed to answer such a question which states that the integral (2.11) is elementary if and only if at least one of the quantities

$$
\frac{p+1}{r}, \quad q, \quad \frac{p+1}{r}+q
$$

is an integer.

Our current work, along the line of [1], is to further explore the insight that may be achieved by the Chebyshev theorem for the integration of (2.5) or (2.10) and present supplementary methods when the problem is out of reach by the theorem. In particular, our main focus will be on the situations where the equation of state is nonlinear.

\section{Perfect Fluids}

In this section we recall some standard materials about perfect fluids as used in cosmology and their representation, when there exits a velocity potential, in terms of a Lagrangian for a scalar field $\psi$ with a shift symmetry $\psi \rightarrow \psi+$ constant.

In $n+1$ spacetime dimensions the energy-momentum tensor is of the form

$$
T^{\mu \nu}=\rho u^{\mu} u^{\nu}+P\left(g^{\mu \nu}+u^{\mu} u^{\nu}\right),
$$

where $\rho$ is energy density, $P$ is pressure and $u^{\mu}$ satisfying $g_{\mu \nu} u^{\mu} u^{\nu}=-1$ is the (timelike) $(n+1)$-velocity of the fluid. So as not to clutter up the formula, we include any cosmological constant in the total energy density and pressure which, in this section, we denote by $\rho$ and $P$ with the subscript $m$ omitted. For simplicity of exposition, in this section, we shall only consider the spacetime dimension $n=3$. The extension to all $n$ is straightforward.

\subsection{Equations of Motion and Thermodynamics}

Define the projection operator $h_{\nu}^{\mu}$ onto the orthogonal complement of the 4 -velocity $u^{\mu}$ by

$$
h_{\nu}^{\mu}=\delta_{\nu}^{\mu}+u^{\mu} u_{\nu}, \quad \Rightarrow \quad h_{\mu \nu}=h_{\nu \mu}, \quad h_{\mu \nu} u^{\nu}=0
$$

Thus for any vector $V^{\mu}$,

$$
V^{\mu}=V_{\|}^{\mu}+V_{\perp}^{\mu}
$$


with

$$
V_{\|}^{\mu}=-u^{\mu}\left(V_{\nu} u^{\nu}\right), \quad V_{\perp}^{\mu}=h_{\nu}^{\mu} V^{\nu},
$$

projecting the equation

$$
\nabla_{\nu} T^{\mu \nu}=0
$$

parallel and perpendicular to $u^{\mu}$ results in

$$
\begin{aligned}
(\rho+P) \nabla_{\mu} u^{\mu}+u^{\mu} \nabla_{\mu} \rho & =0 \\
a^{\mu} & =-\frac{1}{\rho+P} h^{\mu \nu} \nabla_{\nu} P
\end{aligned}
$$

where

$$
a^{\mu}=u^{\nu} \nabla_{\nu} u^{\mu}, \quad \Rightarrow \quad u_{\mu} a^{\mu}=0,
$$

is the acceleration of the fluid. The expansion $\Theta$ of the fluid is defined as

$$
\Theta=\nabla_{\nu} u^{\nu}
$$

and if $V$ is a comoving volume of fluid then $\Theta=\frac{\dot{V}}{V}$.

We now apply the first law of thermodynamics to a volume $V$ of our fluid, assuming no conserved charges or chemical potentials. This states that

$$
\mathrm{d}(\rho V)=T \mathrm{~d}(s V)-P \mathrm{~d} V .
$$

Thus

$$
(\mathrm{d} \rho-T \mathrm{~d} s) V=-(\rho+P-T s) \mathrm{d} V .
$$

Assuming that the fluid is homogeneous, i.e., that $\rho$ and $s$ do not depend upon $V$, we deduce the Gibbs-Duhem relation:

$$
\rho+P=T s .
$$

The quantity $\rho+P$ is the enthalpy density of the fluid and is therefore equal to the product of entropy density $s$ and temperature $T$. Now the first law (3.11) and GibbsDuhem relation (3.12) imply

$$
\mathrm{d} P=s \mathrm{~d} T, \quad \Leftrightarrow \quad \mathrm{d} \rho=T \mathrm{~d} s,
$$

which gives in addition

$$
\frac{\mathrm{d} P}{\rho+P}=\frac{\mathrm{d} T}{T} .
$$

Thus (3.6) implies the conservation of entropy

$$
\nabla_{\mu}\left(s u^{\mu}\right)=0
$$

and the Relativistic Euler equation (3.8) may now be rewritten as

$$
a^{\mu}=-\frac{1}{T} h^{\mu \nu} \nabla_{\nu} T
$$




\subsubsection{Energy Conditions}

The following three energy conditions are important for cosmology:

- The Positive Energy Condition: $T_{\mu \nu} V^{\mu} V^{\mu} \geq 0$ for every future directed timelike vector $V^{\mu}$. In our context this means that $\rho \geq 0$. Thus for a barotropic equation of state such that $P=(\gamma-1) \rho=w \rho$, where $\gamma$ is a constant, positive energy requires $\gamma \geq 1$.

- The Dominant Energy Condition: $-T_{\nu}^{\mu} V^{\nu}$ is a future pointing timelike or null vector. In our context this means that $\rho>|P| \geq 0$. For a barotropic equation of state with positive pressure, dominant energy requires $\gamma \leq 2$. The limiting case $P=\rho$ is called stiff matter and this is satisfied by a massless scalar field (see Subsection 3.5). For negative pressure and positive energy, dominant energy requires $P \geq-\rho$ or $w \geq-1$. The limiting case corresponds to a positive cosmological constant

- The Strong Energy Condition: $\left(T_{\mu \nu}-\frac{1}{2} g_{\mu \nu} T_{\alpha}^{\alpha}\right) V^{\mu} V^{\mu} \geq 0$ for every future directed timelike vector $V^{\mu}$. In our context this means that $\rho+3 P \geq 0$. For a barotropic equation of state, strong energy requires $\gamma \geq \frac{2}{3}$ or $P \geq-\frac{1}{3} \rho$. The limiting case is attained by an isotropic gas of relativistic strings. Incidentally an isotropic gas of relativistic membranes or domain walls has $P=-\frac{2}{3} \rho$ and so violates strong energy by a considerable margin.

Note that for all values of $k$

$$
\frac{\ddot{a}}{a}=-\frac{4 \pi G}{3}(\rho+3 P),
$$

and so acceleration requires a violation of the strong energy equation. Now

$$
\dot{H}=-\frac{12 \pi G}{3}(\rho+P)+\frac{k}{a^{2}}
$$

and so if $k \leq 0, \dot{H} \geq 0$ requires $\rho+P \leq 0$.

\subsubsection{Examples}

If $P=(\gamma-1) \rho$ with $\gamma$ constant then the equation of state is linear and one finds from(3.14) and the Gibbs-Duem relation (3.12)

$$
\rho=\left(\frac{T}{T_{0}}\right)^{\frac{\gamma}{\gamma-1}}, \quad s=\gamma T_{0}^{-\frac{\gamma}{\gamma-1}} T^{\frac{1}{\gamma-1}} .
$$

A simple example of a nonlinear equation of state is provided by the Chaplygin gas for which $P=-\frac{B}{\rho}$ with $B$ constant [7, 8, 9]. One has

$$
\rho+P=\rho-\frac{B}{\rho},
$$


and therefore if $B<\rho^{2}$, the dominant energy condition, i.e. $\rho \geq|P|$, holds, which is consistent with the sound speed $v_{s}=\frac{\partial P}{\partial \rho}=\frac{\sqrt{B}}{\rho}$ being subluminal. The strong energy condition, i.e. $\rho+3 P$ holds as long as $3 B \leq \rho^{2}$.

The temperature is given by

$$
\begin{array}{ll}
\frac{T}{T_{0}}=\sqrt{1-\frac{B}{\rho^{2}}}, \quad \rho^{2}>B, \\
\frac{T}{T_{0}^{\prime}}=\frac{\rho^{2}}{\sqrt{1-\frac{B}{\rho^{2}}}}, \quad \rho^{2}<B .
\end{array}
$$

The dependence on the scale factor is

$$
\rho=\sqrt{B+\frac{C}{a^{6}}}
$$

where $C$ is a constant. If the scale factor is large, this behaves like dark energy plus (if $C>0)$ an admixture of stiff matter. At small scale factors it behaves like dust.

There exists a generalization [9, 10] for which

$$
P=A \rho-\frac{B}{\rho^{\alpha}}
$$

which will be discussed in Subsection 4.1. Note that if $\alpha \geq 0$, then at large $\rho$ the effective coefficient $\gamma=A+1$ and thus $A+1$ will determine which if any energy conditions are satisfied at large $\rho$.

\subsection{Dark Energy}

The simplest interpretation of dark energy is as a cosmological constant term for which

$$
T_{\mu \nu}=-\frac{\Lambda}{8 \pi G} g_{\mu \nu}
$$

This has perfect fluid form with

$$
P_{\text {Dark }}=-\rho_{\text {Dark }}=-\frac{\Lambda}{8 \pi G}=\text { constant. }
$$

Equations (3.6) and (3.8) are unaffected by the replacements

$$
\rho \rightarrow \rho+\rho_{\text {Dark }}, \quad P \rightarrow P-\rho_{\text {Dark }}, \quad g_{\mu \nu} \rightarrow g_{\mu \nu}
$$




\subsection{Stationary Flows}

For a stationary flow

$$
u^{\mu}=\frac{K^{\mu}}{\sqrt{-g_{\sigma v} K^{\sigma} K^{v}}},
$$

where $K^{\mu}$ is a timelike Killing vector field, one has

$$
a^{\mu}=h^{\mu \nu} \nabla_{\nu} \ln \left(\sqrt{-g_{\sigma v} K^{\sigma} K^{v}}\right),
$$

whence

$$
T \sqrt{-g_{\sigma v} K^{\sigma} K^{v}}=\text { constant }
$$

which is Tolman's redshifting law.

\subsection{Irrotational Fluids}

The 4-velocity $u^{\mu}$ is vorticity free if and only if

$$
u \wedge \mathrm{d} u=0
$$

where $u=u_{\mu} \mathrm{d} x^{\mu}$. This implies that

$$
u=Z^{-\frac{1}{2}} \mathrm{~d} \psi
$$

for functions $Z$ and $\psi$. Since $u^{\mu} u_{\mu}=1$ we deduce that

$$
Z=-g^{\mu \nu} \partial_{\mu} \psi \partial_{\nu} \psi
$$

\subsection{Lagrangian Description of Irrotational Perfect Fluids}

Irrotational perfect fluids admit a Lagrangian description in which the Lagrangian is the pressure considered as a function of the velocity potential $\psi$. Such media are often referred to as " $k$-essence". The Lagrangian scalar density is given by

$$
\mathcal{L}=\sqrt{-g} L(Z)
$$

whence

$$
T_{\mu \nu}=-\frac{2}{\sqrt{-g}} \frac{\delta \mathcal{L}}{\delta g^{\mu \nu}}=+2 L_{Z} \partial_{\mu} \psi \partial_{\nu} \psi+g_{\mu \nu} L
$$

Thus

$$
\rho=2 Z L_{z}-L, \quad P=L .
$$

Because of the shift symmetry $\psi \rightarrow \psi+$ constant, a conserved "enthalpy current" is

$$
s^{\mu}=\frac{1}{\sqrt{-g}} \frac{\partial \mathcal{L}}{\partial_{\mu} \psi} .
$$

Some of examples are: 
- Stiff matter aka massless scalar $L=\frac{1}{2} Z, P=\rho$.

- Radiation $L=Z^{2}, P=\frac{1}{3} \rho$.

- Polytrope $L=Z^{\frac{\gamma}{2(\gamma-1)}}, P=(\gamma-1) \rho$.

- Dark Energy $L=$ constant, $P=-\rho$.

- Born-Infeld $L=-\sqrt{1-Z}+1, P=\frac{\rho}{1+\rho}$.

- Chaplygin $L=-\sqrt{1-Z}, P=-\frac{1}{\rho}$.

Note that the last two examples have the same equations of motion but the energy momentum tensor differs by a dark energy term. That is one gets to the Chaplygin case from the Born-Infeld case by the replacements $\rho \rightarrow \rho-1$ and $P \rightarrow P+1$.

The Polytrope equation of motion is the wave equivalent of the $p$-Laplacian. That is

$$
\nabla^{\mu}\left(Z^{\frac{p-2}{2}} \partial_{\mu} \psi\right)=0 .
$$

If we rewrite (3.38) as

$$
\left.\partial_{\mu}\left(\sqrt{-g}\left(-g^{\alpha \beta} \partial_{\alpha} \psi \partial_{\beta} \psi\right)^{\frac{p-2}{2}}\right) g^{\mu \nu} \partial_{\nu} \psi\right)=0,
$$

with $p=\frac{\gamma}{\gamma-1}$, we see that if $p=4$, i.e., for radiation, $\gamma=\frac{4}{3}$, both the Lagrangian and the equation of motion are invariant under conformal rescalings of the metric, i.e., under the replacement $g_{\mu \nu} \rightarrow \Omega^{2} g_{\mu \nu}$.

\subsection{Application to Friedmann-Lemaître Cosmologies}

In this case $\partial_{\mu} \psi=(\dot{\psi}, 0,0,0)$ and so $Z=\dot{\psi}^{2}$. We regard $L(Z)$ as a function of $\dot{\psi}$.

From (3.36) we have

$$
\begin{aligned}
& L(Z)=P(\dot{\psi}), \\
& \rho=\dot{\psi} \frac{\mathrm{d} P}{\mathrm{~d} \dot{\psi}}-P .
\end{aligned}
$$

Now

$$
s^{\mu}=\left(s^{0}, 0,0,0\right),
$$

where

$$
s^{0}=\frac{\mathrm{d} P}{\mathrm{~d} \dot{\psi}},
$$

and conservation of entropy (3.15) gives

$$
a^{3} \frac{\mathrm{d} P}{\mathrm{~d} \dot{\psi}}=\text { constant }
$$

Probably it is best to define say $x=\dot{\psi}$. 
One has

$$
\begin{gathered}
P=P(x), \\
\rho=x \frac{\mathrm{d} P}{\mathrm{~d} x}-P, \\
a^{3} \frac{\mathrm{d} P}{\mathrm{~d} x}=c,
\end{gathered}
$$

where $c$ is a constant. For convenience, we denote $g(x)=\frac{\mathrm{d} P}{\mathrm{~d} x}(x)$ and assume $g$ is invertible. Hence we have $x=g^{-1}\left(c a^{-3}\right)$ and thus

$$
\rho=c a^{-3} g^{-1}\left(c a^{-3}\right)-\int_{0}^{c a^{-3}} g(\xi) \mathrm{d} \xi-P(0) .
$$

This equation relates the energy density $\rho$ to the scale factor $a$ in a complicated way. Nevertheless, many concrete examples are manageable, with or without the Chebyshev theorem. Below are a few of these.

- Set $P=\frac{x^{r+1}}{r+1}$. One finds

$$
\rho=\frac{r}{r+1} x^{r+1}=r P .
$$

From [1] we know that the Friedmann equation is integrable if and only if $\frac{1}{r}$ is among the sequence of numbers

$$
-1, \ldots,-\frac{2}{3},-\frac{5}{9},-\frac{1}{2},-\frac{7}{15},-\frac{4}{9},-\frac{3}{7}, \ldots,-\frac{2}{9},-\frac{1}{5},-\frac{1}{6},-\frac{1}{9}, \frac{1}{3} .
$$

In particular, the case $r=3$, that is

$$
a x=\text { constant }
$$

is well known and corresponds to radiation.

- Chaplygin is

$$
L=-\sqrt{1-x^{2}}, \quad P=-\frac{1}{\rho}
$$

and

$$
a^{3} \frac{x}{\sqrt{1-x^{2}}}=\text { constant. }
$$

A general form of this model will be studied in Section 4.1 .

- Born-Infeld is

$$
L=-\sqrt{1-x^{2}}+1, \quad P=\frac{\rho}{\rho+1},
$$

and

$$
a^{3} \frac{x}{\sqrt{1-x^{2}}}=\text { constant. }
$$

The general situation for this model is thoroughly considered in Section 4.3. 
- A model of current interest is the Bose-Einstein condensate considered by Chavanis [4, 5].

One has

$$
\rho=\sqrt{P_{0} P}+P,
$$

where $P_{0}$ is a constant. We have

$$
\rho=x \frac{\mathrm{d} L}{\mathrm{~d} x}-L,
$$

and hence

$$
\frac{\mathrm{d} L}{2 L+\sqrt{P_{0} L}}=\frac{\mathrm{d} x}{x} .
$$

Thus

$$
L=\frac{1}{4}\left(A x-P_{0}\right)^{2} .
$$

We choose the constant of integration to agree with the Lagrangian for stiff matter so that

$$
P=\frac{1}{4}\left(\sqrt{2} x-P_{0}\right)^{2} .
$$

It follows that

$$
a^{3} \sqrt{P}=\text { constant },
$$

and hence

$$
\rho=\frac{\rho_{\text {dust } 0}}{a^{3}}+\frac{\rho_{\text {stiffmatter } 0}}{a^{6}},
$$

which is a mixture of pressure matter and stiff matter as stated in [4] and a special case of the model investigated in Section 6 .

\section{The Chaplygin Gas and the Born-Infeld Models}

In this section we study the generalized Chaplygin gas and Born-Infeld models. In the former situation, we derive exponentially expanding solutions and obtain their exponential growth rates explicitly in terms of various physical parameters. In particular, these expressions reveal the specific roles played by the linear terms, when nonlinear terms are present, in the equations of state. In the latter situation, we show that, although the Chebyshev theorem is not applicable in general, an integration can still be performed to render explicit solutions.

\subsection{The Chaplygin Gas Model}

The equation of state of a generalized Chaplygin gas model reads [6, 7, 8]

$$
P_{m}=A \rho_{m}-\frac{B}{\rho_{m}^{\alpha}},
$$


where $A, B$ are positive parameters and the exponent $\alpha$ stays in the interval $0 \leq \alpha \leq 1$.

Inserting (4.1) into (2.7) and integrating, we obtain

$$
(1+A) \rho_{m}^{\alpha+1}=C a^{-n(1+A)(\alpha+1)}+B
$$

where $C>0$ is an integration constant. In fact, in our study below, the positivity condition on $A$ can be relaxed to

$$
A>-1
$$

So (4.3) will be the condition on the constant $A$ we observe in the subsequent discussion. Note that the borderline $A=-1$ is known as the Phantom Divide Line (PDL) which is an actively pursued topic [11, 12, 13, 14, 15] in the research on dark matter.

In view of (2.6) and (4.2), we see that (2.5) takes the form

$$
\dot{a}^{2}=\frac{16 \pi G_{n}}{n(n-1)}(1+A)^{-\frac{1}{\alpha+1}} a^{2}\left(C a^{-n(1+A)(\alpha+1)}+B\right)^{\frac{1}{\alpha+1}}+\frac{2 \Lambda}{n(n-1)} a^{2}-k .
$$

In the binomial case, $\Lambda=0, k=0$, integrability by virtue of Chebyshev's theorem is always ensured. As an illustration, we proceed to carry out the integration.

For convenience, we rewrite the Friedmann equation as

$$
\dot{a}^{2}=\beta^{2} a^{2}\left(C a^{-n(1+A)(\alpha+1)}+B\right)^{\frac{1}{\alpha+1}}, \quad \beta^{2}=\frac{16 \pi G_{n}}{n(n-1)}(1+A)^{-\frac{1}{\alpha+1}},
$$

whose integration amounts to computing

$$
I= \pm \frac{1}{\beta} \int a^{-1}\left(C a^{-n(1+A)(\alpha+1)}+B\right)^{-\frac{1}{2(\alpha+1)}} \mathrm{d} a .
$$

Since the constants $B$ and $C$ are positive, we may introduce a new variable $u$ such that

$$
C a^{-n(1+A)(\alpha+1)}+B=u^{2(\alpha+1)} .
$$

Hence we obtain

$$
I=\mp \frac{2}{n(1+A) \beta} \int \frac{u^{2 \alpha}}{u^{2(\alpha+1)}-B} \mathrm{~d} u,
$$

which may be integrated to yield an elementary function if and only if $\alpha$ is rational, say

$$
\alpha=\frac{m_{1}}{m_{2}}, \quad m_{1}, m_{2}=1,2,3, \ldots
$$

Assuming (4.9), we may use

$$
v=B^{-\frac{1}{2\left(m_{1}+m_{2}\right)}} u^{\frac{1}{m_{2}}}
$$

to recast (4.8) into the form

$$
I=\mp \frac{2 m_{2}}{n(1+A) \beta} B^{-\frac{m_{2}}{2\left(m_{1}+m_{2}\right)}} \int \frac{v^{2 m_{1}+m_{2}-1}}{v^{2\left(m_{1}+m_{2}\right)}-1} \mathrm{~d} v,
$$


and then conduct its integration.

If $\alpha$ is irrational, we may approach $\alpha$ by a sequence of rational numbers and use the above integrals to approximate the desired solution.

As an illustration, we consider the classical Chaplygin gas model [9, 10] given simply by the equation of state

$$
P_{m}=A \rho_{m}-\frac{B}{\rho_{m}},
$$

which corresponds to $m_{1}=m_{2}=1$. Hence (4.11) gives us the result

$$
\begin{aligned}
I & =\mp \frac{2}{n(1+A) \beta} B^{-\frac{1}{4}} \int \frac{v^{2}}{v^{4}-1} \mathrm{~d} v \\
& =\mp \frac{1}{n(1+A) \beta} B^{-\frac{1}{4}}\left(\frac{1}{2} \ln \left|\frac{v-1}{v+1}\right|+\arctan v\right)+C_{1},
\end{aligned}
$$

where $C_{1}$ is an integration constant. The big bang universe $a(0)=0$ leads to the solution

$$
2 n(1+A) \beta B^{\frac{1}{4}} t=\ln \left(\frac{\left(B+C a^{-2 n(1+A)}\right)^{\frac{1}{4}}+B^{\frac{1}{4}}}{\left(B+C a^{-2 n(1+A)}\right)^{\frac{1}{4}}-B^{\frac{1}{4}}}\right)-2 \arctan \left(1+\frac{C}{B} a^{-2 n(1+A)}\right)^{\frac{1}{4}}+\pi,
$$

which may further be resolved to yield

$$
a^{2 n(1+A)}(t)=\frac{C}{B\left(\operatorname{coth}^{4}\left[n(1+A) \beta B^{\frac{1}{4}} t+b(t)\right]-1\right)},
$$

where

$$
b(t)=\arctan \left(1+\frac{C}{B} a^{-2 n(1+A)}(t)\right)^{\frac{1}{4}}-\frac{\pi}{2} .
$$

Since $b(t)$ given in (4.16) is a bounded function, we see that the large-time behavior of the scale factor obeys the asymptotic exponential growth law:

$$
a(t) \sim\left(\frac{C}{8 B}\right)^{\frac{1}{2 n(1+A)}} \mathrm{e}^{\beta B^{\frac{1}{4}} t}, \quad t \rightarrow \infty,
$$

which clearly spells out the roles played by various parameters in the model since from the above the asymptotic exponential growth rate is seen to be

$$
\beta B^{\frac{1}{4}}=4\left(\frac{\pi G_{n}}{n(n-1)}\right)^{\frac{1}{2}}\left(\frac{B}{1+A}\right)^{\frac{1}{4}} .
$$

As another illustration, we consider the case when $\alpha=\frac{1}{2}$. Thus, inserting $m_{1}=$ $1, m_{2}=2$ into (4.11), we have

$$
I=\mp \frac{4}{n(1+A) \beta} B^{-\frac{1}{3}} \int \frac{v^{3}}{v^{6}-1} \mathrm{~d} v .
$$


To proceed further, we set $w=v^{2}$ in (4.19) and use the partial fraction decomposition

$$
\frac{w}{w^{3}-1}=\frac{1}{3}\left(\frac{1}{w-1}-\frac{w-1}{w^{2}+w+1}\right)
$$

to arrive at

$$
I=\mp \frac{1}{3 n(1+A) \beta} B^{-\frac{1}{3}}\left(\ln \frac{\left(v^{2}-1\right)^{2}}{v^{4}+v^{2}+1}+2 \sqrt{3} \arctan \frac{2 v^{2}+1}{\sqrt{3}}\right)+C_{1},
$$

where $C_{1}$ is an integration constant. As before, the big bang solution reads

$$
\begin{aligned}
3 n(1+A) \beta B^{\frac{1}{3}} t= & \ln \left(\frac{\left[1+\frac{C}{B} a^{-\frac{3}{2} n(1+A)}\right]^{\frac{2}{3}}+\left[1+\frac{C}{B} a^{-\frac{3}{2} n(1+A)}\right]^{\frac{1}{3}}+1}{\left(\left[1+\frac{C}{B} a^{-\frac{3}{2} n(1+A)}\right]^{\frac{1}{3}}-1\right)^{2}}\right) \\
& -2 \sqrt{3} \arctan \frac{1}{\sqrt{3}}\left(2\left[1+\frac{C}{B} a^{-\frac{3}{2} n(1+A)}\right]^{\frac{1}{3}}+1\right)+\sqrt{3} \pi .
\end{aligned}
$$

Resolving this relation, we obtain

$$
\begin{aligned}
& \left(1+\frac{C}{B} a^{-\frac{3}{2} n(1+A)}(t)\right)^{\frac{1}{3}}= \\
& \frac{2+\mathrm{e}^{-3 n(1+A) \beta B^{\frac{1}{3}} t+2 b(t)}+\mathrm{e}^{-\frac{3}{2} n(1+A) \beta B^{\frac{1}{3}} t+b(t)}\left(12-3 \mathrm{e}^{-3 n(1+A) \beta B^{\frac{1}{3}} t+2 b(t)}\right)^{\frac{1}{2}}}{2-\mathrm{e}^{-3 n(1+A) \beta B^{\frac{1}{3}} t+2 b(t)}},
\end{aligned}
$$

where

$$
b(t)=\sqrt{3} \arctan \frac{1}{\sqrt{3}}\left(2\left[1+\frac{C}{B} a^{-\frac{3}{2} n(1+A)}(t)\right]^{\frac{1}{3}}+1\right)-\frac{\sqrt{3}}{2} \pi
$$

is a bounded function. Therefore we obtain the asymptotic exponential growth law as before:

$$
a(t) \sim\left(\frac{C}{3 \sqrt{3} B}\right)^{\frac{2}{3 n(1+A)}} \mathrm{e}^{\beta B^{\frac{1}{3}} t}, \quad t \rightarrow \infty .
$$

Thus we also get the asymptotic exponential growth rate as follows:

$$
\beta B^{\frac{1}{3}}=4\left(\frac{\pi G_{n}}{n(n-1)}\right)^{\frac{1}{2}}\left(\frac{B}{1+A}\right)^{\frac{1}{3}} .
$$

For later convenience, we use (4.7) and (4.10) to express the dependence of $a$ and $v$ directly as

$$
v=B^{-\frac{1}{2\left(m_{1}+m_{2}\right)}}\left(C a^{-n(1+A) \frac{\left(m_{1}+m_{2}\right)}{m_{2}}}+B\right)^{\frac{1}{2\left(m_{1}+m_{2}\right)}}
$$


Thus the big bang initial condition $a(0)=0$ with $A>-1$ leads to

$$
v(0)=\infty
$$

Hence, from (4.11) and (4.28), we obtain the expanding solution

$$
\left(\frac{n(1+A) \beta}{2 m_{2}} B^{\frac{m_{2}}{2\left(m_{1}+m_{2}\right)}}\right) t=\int_{v(t)}^{\infty} \frac{v^{2 m_{1}+m_{2}-1}}{v^{2\left(m_{1}+m_{2}\right)}-1} \mathrm{~d} v, \quad t>0,
$$

which monotonically climbs to the limiting level $v=1$ as $t \rightarrow \infty$.

As yet another example, we take $m_{1}=1$ and $m_{2}=3$ (i.e., $\alpha=\frac{1}{3}$ ). Thus (4.29) yields

$$
\begin{aligned}
\frac{8}{3} n(1+A) \beta B^{\frac{3}{8}} t= & \ln \left(\frac{v+1}{v-1}\right)^{2}+4 \arctan v+\sqrt{2} \ln \left(\frac{v^{2}-\sqrt{2} v+1}{v^{2}+\sqrt{2} v+1}\right) \\
& -2 \sqrt{2}(\arctan [\sqrt{2} v+1]+\arctan [\sqrt{2} v-1]) \\
& +2(\sqrt{2}-1) \pi, \quad v=v(t)>1, \quad t>0 .
\end{aligned}
$$

Since all the terms except the first one on the right-hand side of (4.30) stay bounded, we have

$$
v(t) \sim \operatorname{coth}\left(\frac{2}{3} n(1+A) \beta B^{\frac{3}{8}} t\right), \quad t \rightarrow \infty .
$$

In view of (4.27) and (4.31), we arrive at

$$
a(t) \sim\left(\frac{C}{16 B}\right)^{\frac{3}{4 n(1+A)}} \mathrm{e}^{\beta B^{\frac{3}{8}} t}, \quad t \rightarrow \infty .
$$

Consequently, we get the asymptotic exponential growth rate as before:

$$
\beta B^{\frac{3}{8}}=4\left(\frac{\pi G_{n}}{n(n-1)}\right)^{\frac{1}{2}}\left(\frac{B}{1+A}\right)^{\frac{3}{8}} .
$$

\subsection{A Universal Formula for Asymptotic Exponential Growth Rate and Cosmological Consequences}

With the explicit knowledge on exact solutions described earlier as stated in (4.17), (4.25), and (4.30), we are able to derive such precise formulas as (4.18), (4.26), and (4.33) for the exponential growth rate of the scale factor. In these examples, it is interesting, and perhaps surprising, to see that, in the context of the Chaplygin gas model, the linear part of the equation of state switched on by the coefficient $A$ in (4.1) also contributes to the asymptotic exponential growth rate of the scale factor, as seen in the examples (4.18), (4.26), and (4.33) in the previous subsection, which follow a clear pattern of the following characteristics:

- The growth rate contains a common factor which depends on the Newton constant $G_{n}$ and spatial dimensionality $n$ only. 
- The growth rate is proportional to some power $\gamma$ (say) of the common ratio $\frac{B}{1+A}$ composed from the linear and nonlinear coupling constants $A$ and $B$ in (4.1). In (4.18), (4.26), and (4.33), we have $\gamma=\frac{1}{4}, \frac{1}{3}$, and $\frac{3}{8}$, corresponding to $\alpha=1, \frac{1}{2}$, and $\frac{1}{3}$, in (4.1), respectively.

Inspired by the above examples, in this subsection, we will derive a universal formula for the asymptotic exponential growth rate of the scale factor for the generalized Chaplygin model (4.1), regardless whether $\alpha$ is rational. We then elaborate on some applications of this general formula to the study of dark matter.

For convenience, we use the new variable

$$
w=B^{-\frac{1}{2(\alpha+1)}} u
$$

in (4.8). Thus (4.7) gives us

$$
w=\left(\frac{C}{B} a^{-n(1+A)(\alpha+1)}+1\right)^{\frac{1}{2(\alpha+1)}} .
$$

Therefore the boundary condition consisting of $a(0)=0$ and $a(\infty)=\infty$ becomes

$$
w(0)=\infty, \quad w(\infty)=1 .
$$

From (4.8) and (4.36), we arrive at

$$
\frac{1}{2} n(1+A) \beta B^{\frac{1}{2(\alpha+1)}} t=\int_{w(t)}^{\infty} \frac{w^{2 \alpha}}{w^{2(\alpha+1)}-1} \mathrm{~d} w, \quad w(t)>1 .
$$

Since $w^{2(\alpha+1)}-1$ is approximated by $2(\alpha+1)(w-1)$ for $w$ near 1 , we have

$$
\int_{w(t)}^{\infty} \frac{w^{2 \alpha}}{w^{2(\alpha+1)}-1} \mathrm{~d} w=\frac{1}{2(\alpha+1)} \int_{w(t)}^{w\left(t_{0}\right)} \frac{1}{w-1} \mathrm{~d} w+\mathrm{O}(1)
$$

where $t>t_{0}>0$ and $\mathrm{O}(1)$ denotes a bounded quantity. Combining (4.37) and (4.38), we obtain

$$
w(t)=1+\left(w\left(t_{0}\right)-1\right) \mathrm{e}^{-n(1+A)(\alpha+1) \beta B^{\frac{1}{2(\alpha+1)}} t+\mathrm{o}(1)}, \quad t>t_{0} .
$$

Using (4.35) and (4.39), we have the asymptotic growth pattern

$$
a(t) \sim C_{0} \mathrm{e}^{\beta B^{\frac{1}{2(\alpha+1)}} t}, \quad t>t_{0},
$$

where $C_{0}>0$ is some constant. Consequently we see that the asymptotic exponential growth rate of the scale factor $a$ is given by the universal formula

$$
\beta B^{\frac{1}{2(\alpha+1)}}=4\left(\frac{\pi G_{n}}{n(n-1)}\right)^{\frac{1}{2}}\left(\frac{B}{1+A}\right)^{\frac{1}{2(\alpha+1)}},
$$


which recovers the formulas (4.18), (4.26), and (4.33) when $\alpha=1, \frac{1}{2}$, and $\frac{1}{3}$, respectively, and exactly confirms the characteristics described at the beginning of this subsection.

It will be interesting to compare the above result with the situation when the nonlinear term in the Chaplygin law is turned off, by setting $B=0$ in (4.1). Now the conservation law (2.7) leads to

$$
\rho=\rho_{0} a^{-n(1+A)}, \quad A \in \mathbb{R} .
$$

Hence, integrating the Friedmann equation (for $\Lambda=0, k=0$ ), we have the (well-known) expanding solution:

$$
a(t)= \begin{cases}\left(4 \pi G_{n} \rho_{0}\left[\frac{n}{n-1}\right](1+A)^{2}\right)^{\frac{1}{n(1+A)}} t^{\frac{2}{n(1+A)}}, & A>-1, t>0, a(0)=0 \\ a(0) \mathrm{e}^{4 \sqrt{\pi G_{n} \rho_{0} / n(n-1)} t}, & A=-1, t>0, a(0)>0 \\ \left(a^{\frac{n(1+A)}{2}}(0)-4\left[\frac{\pi G_{n} \rho_{0}}{n(n-1)}\right]^{\frac{1}{2}} t\right)^{\frac{2}{n(1+A)}}, & A<-1, t>0, a(0)>0 .\end{cases}
$$

So, when the nonlinear matter is absent and linear matter is that of a conventional type, $A>-1$, the scale factor of a big-bang universe fails to grow exponentially but instead follows a power-function type growth law, and, when $A \leq-1$, the big-bang solution with $a(0)=0$ is not allowed and the universe may only evolve from a positive value of the scale factor, $a(0)>0$. In this latter situation, the condition $A<-1$ corresponds to a hypothetical model called the phantom-energy universe [16, 17] where the solution blows up at finite time

$$
t_{0}=\frac{a^{\frac{n(1+A)}{2}}(0)}{4\left(\frac{\pi G_{n} \rho_{0}}{n(n-1)}\right)^{\frac{1}{2}}},
$$

known as the Big Rip [18, 19] epoch. The limiting situation, $A=-1$, is the PDL referred to earlier, where the exponential expansion rate of the scale factor may assume arbitrarily large value, depending on the value of $\rho_{0}$.

On the other hand, the universal formula (4.41) indicates that, within the context of the generalized Chaplygin fluid (4.1), a conventional $(A>-1)$ linear term alone does not give rise to exponential growth but it plays a role in exponential growth when a nonlinear term is present, no matter how weak this nonlinear matter is, and that, this linear contribution becomes significant near the PDL limit. That is, the asymptotic exponential expansion rate of the scale factor may assume arbitrarily large values for $A>-1$ and $A$ stays near -1 . In other words, a tiny presence of the Chaplygin matter would switch on the presence of dark matter which becomes significant near the PDL limit.

\subsection{The Born-Infeld Type Fluid Model}

In this subsection, we shall consider a Born-Infeld type fluid whose equation of state is of the form

$$
P_{m}=\frac{\rho_{m}}{\rho_{m}+1}
$$


As we saw in Section [3, there is a close relation between the Born-Infeld and the classical Chaplygin gas models. The two Lagrangians differ by a constant which adds a cosmological or dark energy term. Thus if $\tilde{\rho}_{m}=\rho_{m}+1$ and $\tilde{P}_{m}=P_{m}-1$ we find

$$
\tilde{P}_{m}=-\frac{1}{\tilde{\rho}_{m}}
$$

However, the Born-Infeld equation of state, unlike the Chaplygin gas, satisfies both the dominant and strong energy conditions for all values of the energy density.

Inserting (4.45) into (2.7) gives us the following dependence relation between the mass density and the scale factor:

$$
\rho_{m}=-1+\sqrt{1+C_{0} a^{-2 n}}
$$

where $C_{0}>0$ is a constant. Hence, in view of (2.6) , the Friedmann equation (2.5) becomes

$$
H^{2}=\frac{16 \pi G_{n}}{n(n-1)}\left(-1+\sqrt{1+C_{0} a^{-2 n}}+\frac{\Lambda}{8 \pi G_{n}}\right)-\frac{k}{a^{2}} .
$$

First, we see that, in order to be able to utilize the Chebyshev theorem for integration of (4.48), we have to assume $k=0$ and

$$
\Lambda=8 \pi G_{n}
$$

In such a situation, the Friedmann equation (4.48) is reduced into (4.5) which corresponds to the Chaplygin gas model (4.1) with $\alpha=1, A=0, B=1$ and whose solution is given in (4.13) with $C=C_{0}$ in (4.7). Thus various solutions of specific interests may be written down immediately. For example, the solution satisfying $a(0)=0$ is given by

$$
\ln \left|\frac{u-1}{u+1}\right|+2 \arctan u=\pi \mp 8 \sqrt{\frac{n \pi G_{n}}{n-1}} t, \quad u=\left(1+C_{0} a^{-2 n}\right)^{\frac{1}{4}} .
$$

Next, we note that, when (4.49) is not fulfilled and, so, the Chebyshev theorem fails to be directly applicable, we can still integrate (4.48) at $k=0$. For this purpose, we set

$$
\alpha=\frac{\Lambda}{8 \pi G_{n}}-1, \quad \beta^{2}=\frac{16 \pi G_{n}}{n(n-1)},
$$

and rewrite (4.48) as

$$
(\dot{a})^{2}=\beta^{2} a^{2}\left(\alpha+\sqrt{1+C_{0} a^{-2 n}}\right),
$$

whose integration reads

$$
I=\int a^{-1}\left(\alpha+\sqrt{1+C_{0} a^{-2 n}}\right)^{-\frac{1}{2}} \mathrm{~d} a= \pm \beta t .
$$

Obviously we cannot apply the Chebyshev theorem as before. Nevertheless, we may set $1+C_{0} a^{-2 n}=u^{2}$ so that $a=C_{0}^{\frac{1}{2 n}}\left(u^{2}-1\right)^{-\frac{1}{2 n}}$ to convert the left-hand side of (4.53) into

$$
I=-\frac{1}{n} \int u\left(u^{2}-1\right)^{-1}(\alpha+u)^{-\frac{1}{2}} \mathrm{~d} u \text {. }
$$


Now set $\alpha+u=w^{2}$ or $u=w^{2}-\alpha$. Then (4.54) becomes

$$
\begin{aligned}
I & =-\frac{2}{n} \int \frac{w^{2}-\alpha}{\left(w^{2}-\alpha\right)^{2}-1} \mathrm{~d} w \\
& =-\frac{1}{n} \int\left(\frac{1}{w^{2}-(\alpha+1)}+\frac{1}{w^{2}-(\alpha-1)}\right) \mathrm{d} w
\end{aligned}
$$

whose integration up to an additive integration constant is readily computed to give us the results:

$$
I= \begin{cases}-\frac{1}{n}\left(\frac{1}{2 \sqrt{1+\alpha}} \ln \left|\frac{w-\sqrt{1+\alpha}}{w+\sqrt{1+\alpha}}\right|+\frac{1}{\sqrt{1-\alpha}} \arctan \frac{w}{\sqrt{1-\alpha}}\right), & |\alpha|<1, \\ -\frac{1}{n}\left(\frac{1}{2 \sqrt{2}} \ln \left|\frac{w-\sqrt{2}}{w+\sqrt{2}}\right|-\frac{1}{w}\right), & \alpha=1, \\ -\frac{1}{2 n}\left(\frac{1}{\sqrt{1+\alpha}} \ln \left|\frac{w-\sqrt{1+\alpha}}{w+\sqrt{1+\alpha}}\right|+\frac{1}{\sqrt{\alpha-1}} \ln \left|\frac{w-\sqrt{\alpha-1}}{w+\sqrt{\alpha-1}}\right|\right), & \alpha>1, \\ -\frac{1}{n}\left(\frac{1}{\sqrt{2}} \arctan \frac{w}{\sqrt{2}}-\frac{1}{w}\right), & \alpha=-1, \\ -\frac{1}{n}\left(\frac{1}{\sqrt{-(\alpha+1)}} \arctan \frac{w}{\sqrt{-(\alpha+1)}}+\frac{1}{\sqrt{1-\alpha}} \arctan \frac{w}{\sqrt{1-\alpha}}\right), & \alpha<-1 .\end{cases}
$$

Thus, in short, we conclude that the Born-Infeld fluid model (4.45) allows an exact integration when $k=0$ for any value of the cosmological constant, although it lies beyond the scope of the Chebyshev theorem.

To end this section, we examine the case $\alpha>1$ closely as an illustrative example. It is readily seen that the big bang solution, with $a(0)=0$ and $a(t)>0$ for $t>0$, is allowed which is given by

$$
\begin{aligned}
2 n \beta t= & \frac{1}{\sqrt{1+\alpha}} \ln \left|\frac{\sqrt{\alpha+\sqrt{1+C_{0} a^{-2 n}}}+\sqrt{1+\alpha}}{\sqrt{\alpha+\sqrt{1+C_{0} a^{-2 n}}}-\sqrt{1+\alpha}}\right| \\
& +\frac{1}{\sqrt{\alpha-1}} \ln \left|\frac{\sqrt{\alpha+\sqrt{1+C_{0} a^{-2 n}}}+\sqrt{\alpha-1}}{\sqrt{\alpha+\sqrt{1+C_{0} a^{-2 n}}}-\sqrt{\alpha-1}}\right| .
\end{aligned}
$$

Thus, in the limit $a(t) \rightarrow \infty$ as $t \rightarrow \infty$, since the second term on the right-hand side of the above is bounded, we have the asymptotic formula:

$$
\begin{aligned}
a(t) & \sim\left(\frac{C_{0}}{8(1+\alpha)}\right)^{\frac{1}{2 n}} \mathrm{e}^{\beta \sqrt{1+\alpha} t} \\
& =\left(\frac{\pi G_{n} C_{0}}{\Lambda}\right)^{\frac{1}{2 n}} \mathrm{e}^{\sqrt{\frac{2 \Lambda}{n(n-1)}} t}, \quad t \rightarrow \infty .
\end{aligned}
$$

It is interesting to note that the Newton constant is absent from the exponent of the asymptotic growth law. 


\section{Multiple Fluids}

In this section we study the integrability problem for several multiple fluid models in view of the Chebyshev theorem. In a two-term energy density model, we give an example of explicit solution that obeys a linear asymptotic growth law. In a two-fluid model, we show how to use the information from integration to obtain periodic solutions linking big-bang to big crunch epochs. In a trinomial Friedmann equation model, we find exponentially expanding solutions.

\subsection{A Two-Term Energy Density Model}

Consider now the classical Friedmann equation in three-spatial dimensions so that (2.5) assumes the form

$$
H^{2}=\frac{8 \pi G}{3} \rho-\frac{k}{a^{2}}
$$

In $[20,21]$ the energy density is taken to enjoy the general decomposition

$$
\rho=\sum_{n=-\infty}^{\infty} \rho_{n}^{+} a^{-n}+\sum_{m=-\infty}^{\infty} \rho_{m}^{-} a^{-m}
$$

where the quantities $\rho_{n}^{+}$'s are positive constants and, $\rho_{m}^{-}$'s, negative. Following [20, 21], we study the simple situation that only two terms in (5.2) are present and $k$ is set to zero so that the Friedmann equation becomes

$$
\frac{\dot{a}^{2}}{H_{0}^{2}}=\Omega_{n}^{+} a^{2-n}+\Omega_{m}^{-} a^{2-m},
$$

where $\Omega_{n}^{+}>0$ and $\Omega_{m}^{-}<0$ are the normalized positive and negative energy density coefficients and $H_{0}>0$ is the Hubble parameter at a time when $a=1$, although our study here does not need to be confined within such a sign condition regarding the quantities $\Omega_{n}^{+}$and $\Omega_{m}^{-}$. Hence, from (5.3), we arrive at the integral

$$
I=\frac{1}{H_{0}} \int a^{\frac{n}{2}-1}\left(\Omega_{n}^{+}+\Omega_{m}^{-} a^{n-m}\right)^{-\frac{1}{2}} \mathrm{~d} a= \pm t .
$$

When $m=n$, (5.4) is trivially computed. Thus, in the sequel we assume $m \neq n$. In view of the Chebyshev theorem we see that (5.4) may be integrated if and only if $m, n(m \neq n)$ satisfy one of the following conditions:

$$
\begin{aligned}
n & =\frac{2 N}{2 N-1} m, \quad N=0, \pm 1, \pm 2, \ldots, \\
m & =\frac{2 N}{2 N+1} n, \quad N=0, \pm 1, \pm 2, \ldots
\end{aligned}
$$

In particular, if $m=0$ or $n=0$, integrability is ensured for any integer $n$ or $m$. 
As an illustration, we show how to integrate (5.4) under the condition (5.5). In fact, in this case, (5.5) may be rewritten as

$$
I=\frac{1}{H_{0}} \int a^{\frac{m N}{2 N-1}-1}\left(\Omega_{n}^{+}+\Omega_{m}^{-} a^{\frac{m}{2 N-1}}\right)^{-\frac{1}{2}} \mathrm{~d} a .
$$

Now set

$$
\Omega_{n}^{+}+\Omega_{m}^{-} a^{\frac{m}{2 N-1}}=u^{2}
$$

which leads to the inverse relation

$$
a=\left(\frac{u^{2}}{\Omega_{m}^{-}}-\frac{\Omega_{n}^{+}}{\Omega_{m}^{-}}\right)^{\frac{2 N-1}{m}}
$$

so that (5.7) reduces into the integral

$$
I=\frac{2(2 N-1)}{m H_{0}\left(\Omega_{m}^{-}\right)^{N}} \int\left(u^{2}-\Omega_{n}^{+}\right)^{N-1} \mathrm{~d} u,
$$

that allows a recursive computation with the substitution $u=\sqrt{\Omega_{n}^{+}} \sec \theta$, say. Note that, from (5.8), we have the restriction

$$
\Omega_{n}^{+}-u^{2}=\left|\Omega_{m}^{-}\right| a^{\frac{m}{2 N-1}} \geq 0
$$

for the range of the variable $u$.

After the above general discussion, we consider a concrete situation with $m=3, n=$ $2, N=-1$. Thus, from integrating (5.10), we get

$$
\pm \frac{H_{0}}{\left|\Omega_{3}^{-}\right|} t=-\frac{u}{\Omega_{2}^{+}\left(u^{2}-\Omega_{2}^{+}\right)}+\left(\Omega_{2}^{+}\right)^{-\frac{3}{2}} \operatorname{arctanh}\left(\frac{u}{\sqrt{\Omega_{2}^{+}}}\right)+C,
$$

where $C$ is an integration constant. On the other hand, since $N=-1$, we need to strengthen (5.11) into

$$
\Omega_{2}^{+}-u^{2}=\left|\Omega_{3}^{-}\right| a^{-1}>0 .
$$

In particular, we have the lower bound

$$
a(0) \geq \frac{\left|\Omega_{3}^{-}\right|}{\Omega_{2}^{+}}
$$

for the initial value of the scale factor so that a big-bang universe is not allowed. Fix the constant $C$ in (5.12) with the initial value of the scale factor at $t=0$ satisfying (5.14). Choose the plus sign in (5.12) representing an expanding universe. Then, from (15.12), we see that

$$
\lim _{t \rightarrow \infty} u(t)=\sqrt{\Omega_{2}^{+}}
$$


or $a(t) \rightarrow \infty$ as $t \rightarrow \infty$. Since the first term in (5.12) is dominant near $u=\sqrt{\Omega_{2}^{+}}$, we arrive, in view of (5.13), at the asymptotic linear growth law:

$$
a(t) \sim \sqrt{\Omega_{2}^{+}} H_{0} t, \quad t \rightarrow \infty
$$

It is interesting, and perhaps surprising, to note that $\Omega_{3}^{-}$makes no appearance here.

Another interesting example that fits our study is the Friedmann equation

$$
H^{2}=\frac{8 \pi G}{3}\left(\rho_{d}+\rho_{b}+\rho_{\gamma}\right)
$$

considered in [22] in the context of the so-called geometrothermodynamics (GTD) [23], where $\rho_{d}$ is the GTD dark matter density given in terms of the scale factor $a$ by

$$
\rho_{d}=\rho_{d 0}\left(\mathcal{A} a^{-3(\alpha-\beta)}+(1-\mathcal{A}) a^{-3(1+\alpha)}\right)^{\frac{1}{1+\alpha}}, \quad \mathcal{A}>0, \alpha, \beta \in \mathbb{R},
$$

and $\rho_{b}=\rho_{b 0} a^{-3}$ and $\rho_{\gamma}=\rho_{\gamma 0} a^{-4}$ are the baryonic and relativistic matter densities. Thus, when some terms in these densities are absent so that the integration of (5.17) is of a binomial type, the Chebyshev theorem becomes applicable. Here we discuss such a situation.

For simplicity, we assume the baryonic and relativistic matter densities in (5.17) are absent. Hence we arrive at the integration

$$
\pm 2 \sqrt{\frac{2 \pi G}{3}} t=I_{\alpha, \beta} \equiv \int a^{\frac{1}{2}}\left((1-\mathcal{A})+\mathcal{A} a^{3(1+\beta)}\right)^{-\frac{1}{2(1+\alpha)}} \mathrm{d} a .
$$

Thus, in view of the Chebyshev theorem, we see that, when $\alpha, \beta$ are rational numbers, $I_{\alpha, \beta}$ is elementary if and only if one of the following three quantities

$$
\frac{1}{2(1+\alpha)}, \quad \frac{1}{2(1+\beta)}, \quad \frac{1}{2(1+\alpha)}-\frac{1}{2(1+\beta)}=\frac{\beta-\alpha}{2(1+\alpha)(1+\beta)}
$$

is an integer. For example, when $\alpha=\beta=-\frac{3}{2}$, we have

$$
I_{-\frac{3}{2},-\frac{3}{2}}=\mathcal{A} \ln a+\frac{2}{3}(1-\mathcal{A}) a^{\frac{3}{2}}+C
$$

where $C$ is an integration constant. Hence we see that the big bang solution, $a(0)=0$, is not allowed, and when $\mathcal{A}<1$ the scale factor obeys a power law growth pattern and when $\mathcal{A}=1$ an exponential pattern.

\subsection{A Two-Fluid Model}

Consider the equation of state of a two-fluid model

$$
\rho_{m}=\frac{2 R}{a^{3}}+\frac{A^{2}-R^{2}}{a^{4}},
$$


where $A, R>0$ are constants and both cases $A>R$ and $A<R$ are of cosmological interest. If $n=3$, the first term corresponds to pressure free matter and the second to radiation. We are interested in a closed universe with vanishing cosmological constant. In that case the Friedmann equation reads

$$
(\dot{a})^{2}=\frac{16 \pi G_{n}}{n(n-1)} \frac{\left(A^{2}-R^{2}+2 R a-B_{n} a^{2}\right)}{a^{2}},
$$

where

$$
B_{n}=\frac{n(n-1)}{16 \pi G_{n}}
$$

The necessary condition for (5.23) to be meaningful, that is, the right-hand side of (5.23) is positive for some values of $a$, is

$$
R^{2}<A^{2}+\frac{R^{2}}{B_{n}}
$$

which guarantees that the quadratic function

$$
q(a)=A^{2}-R^{2}+2 R a-B_{n} a^{2}
$$

has two real roots, which are

$$
a_{ \pm}=\frac{R}{B_{n}} \pm \sqrt{\left(\frac{R}{B_{n}}\right)^{2}+\frac{1}{B_{n}}\left(A^{2}-R^{2}\right)} .
$$

If $A>R$, then $a_{+}>0$ and $a_{-}<0$; if $A \leq R$, then $a_{+}>a_{-} \geq 0$. In either case, we may rewrite (5.23) as

$$
(\dot{a})^{2}=\frac{\left(a-a_{-}\right)\left(a_{+}-a\right)}{a^{2}}
$$

where $a$ stays in the interval $0 \leq a \leq a_{+}$if $A>R$; in $a_{-} \leq a \leq a_{+}$if $A \leq R$, which formally leads to its integration

$$
I=\int a\left(\left(a-a_{-}\right)\left(a_{+}-a\right)\right)^{-\frac{1}{2}} \mathrm{~d} a= \pm t
$$

Setting $u=a-a_{-}$, we have

$$
\begin{aligned}
\left(\sqrt{\left(\frac{R}{B_{n}}\right)^{2}+\frac{1}{B_{n}}\left(A^{2}-R^{2}\right)}-\frac{R}{B_{n}}\right) & =-a_{-} \leq u \leq a_{+}-a_{-} \\
& =2 \sqrt{\left(\frac{R}{B_{n}}\right)^{2}+\frac{1}{B_{n}}\left(A^{2}-R^{2}\right)}
\end{aligned}
$$

and we can rewrite the integral $I$ on the left-hand side of (5.29) as

$$
I=\int u^{\frac{1}{2}}\left(\left(a_{+}-a_{-}\right)-u\right)^{-\frac{1}{2}} \mathrm{~d} u+a_{-} \int u^{-\frac{1}{2}}\left(\left(a_{+}-a_{-}\right)-u\right)^{-\frac{1}{2}} \mathrm{~d} u \equiv I_{1}+I_{2} .
$$


It is easily seen that the Chebyshev theorem implies that both $I_{1}$ and $I_{2}$ are integrable to yield elementary functions. Carrying out the integrations, we obtain

$$
I=\frac{1}{2}\left(a_{+}+a_{-}\right) \arctan \left(\frac{\left(a-a_{+}\right)+\left(a-a_{-}\right)}{2 \sqrt{\left(a_{+}-a\right)\left(a-a_{-}\right)}}\right)-\sqrt{\left(a_{+}-a\right)\left(a-a_{-}\right)}+C,
$$

where $C$ is an integration constant. If $A>R$, the big bang solution, $a(0)=0$, is allowed; if $A \leq R$, the initial value of the scale factor has to satisfy $a(0) \geq a_{-}$. These fix the constant $C$.

Below we consider the big bang solution with the assumption $A>R$. Thus $C$ in (5.32) is given as

$$
C \equiv C_{0}=\sqrt{a_{+}\left(-a_{-}\right)}+\frac{1}{2}\left(a_{+}+a_{-}\right) \arctan \left(\frac{a_{+}+a_{-}}{2 \sqrt{a_{+}\left(-a_{-}\right)}}\right) .
$$

It is clear that the solution increases monotonically until it hits $a=a_{+}$when $t$ becomes

$$
t=t_{s}=C_{0}+\frac{\pi}{4}\left(a_{+}+a_{-}\right)
$$

From (5.28) we find $\dot{a}\left(t_{s}\right)=0$. Furthermore, differentiating (5.28) and taking the limit $t \rightarrow t_{s}^{-}$, we have

$$
\ddot{a}\left(t_{s}\right)=-\frac{\left(a_{+}-a_{-}\right)}{2 a_{+}^{2}}<0 .
$$

This indicates that the solution starts to decrease beyond $t_{s}$. In fact, if we use $a(t)$ to denote the solution obtained above in the time interval $0 \leq t \leq t_{s}$, the solution of (5.28) that continues to evolve beyond $t_{s}$ is given by

$$
a\left(2 t_{s}-t\right), \quad t_{s} \leq t \leq 2 t_{s}
$$

as can be verified directly. At the time $2 t_{s}$, the solution vanishes again and then repeats the same cycle. That is, we have obtained a periodic solution which starts from $a=0$ at $t=0$, climbs to its maximum height $a_{+}$at half period $\frac{1}{2} t_{p}=t_{s}$, and then starts to descend steadily until $a=0$ at a full period $t_{p}=2 t_{s}$. The quantity $t_{s}$ may be called time of maximum expansion. In fact $t_{s}$ is a moment of time symmetry since the solution curve of the scale factor against time is clearly symmetric about its time of maximum expansion. Typically such solutions expand from a "Big-Bang" and recollapse to a "Big Crunch". In the case of a negative cosmological constant, the Big-Bang is a coordinate singularity and the solution may be extended to a periodic solution. In the case of a barotropic equation of state, both the Big Bang and the Big Crunch are singular there is no unambiguous periodic continuation.

For the record, we can explicitly rewrite in view of (5.27), (5.33), and (5.34) the period 
$t_{p}$ just obtained as follows:

$$
\begin{aligned}
t_{p} & =2 C_{0}+\frac{\pi}{2}\left(a_{+}+a_{-}\right) \\
& =8\left(\sqrt{\frac{\pi G_{n}\left(A^{2}-R^{2}\right)}{n(n-1)}}+\frac{2 \pi^{2} G_{n} R}{n(n-1)}+\frac{4 \pi G_{n} R}{n(n-1)} \arctan \left[\frac{4 R \sqrt{\pi G_{n}}}{\sqrt{n(n-1)\left(A^{2}-R^{2}\right)}}\right]\right) .
\end{aligned}
$$

Thus we have arrived at a comprehensive and accurate understanding of the evolution of the scale factor in the two-fluid closed-universe model with respect to cosmic time.

The problem of this section motivates us to study the equation of state for the situation that the mass density and scale factor are related simply by

$$
\rho_{m}=\frac{A}{a^{r}}+\frac{B}{a^{s}}, \quad r \neq s,
$$

where $A, B, r, s$ are constants. Using (5.38) in the conservation law (2.7) which may be rewritten in the form

$$
P_{m}=-\rho_{m}-\frac{a}{n} \frac{\mathrm{d} \rho_{m}}{\mathrm{~d} a}
$$

we find

$$
P_{m}=\frac{A}{a^{r}}\left(\frac{r}{n}-1\right)+\frac{B}{a^{s}}\left(\frac{s}{n}-1\right) .
$$

We are interested in the case when $P_{m}$ is simply a power function of $a$. Thus, without loss of generality, we may assume

$$
r=n
$$

which leads us to arrive at

$$
a^{s} P_{m}=B\left(\frac{s}{n}-1\right), \quad s \neq n
$$

and the equation of state

$$
\rho_{m}=\frac{n P_{m}}{(s-n)}+A\left(\frac{n P_{m}}{B(s-n)}\right)^{\frac{n}{s}} .
$$

\subsection{A Trinomial Friedmann Equation}

We consider the trinomial Friedmann equation with $k=1$ and a nonzero cosmological constant $\Lambda$ of the form [24]

$$
H^{2}=\frac{16 \pi G_{n}}{n(n-1)} \rho_{m}+\frac{2 \Lambda}{n(n-1)}-\frac{1}{a^{2}},
$$

where the mass density $\rho_{m}$ is taken to obey the specific relation

$$
\rho_{m}=\frac{C_{1}}{a^{n+1}},
$$


in which $C_{1}$ is a constant, so that an integration of the equation leads to

$$
\pm t=I_{n}=\int\left(-1+\frac{2 \Lambda}{n(n-1)} a^{2}+\frac{16 \pi G_{n} C_{1}}{n(n-1)} a^{-(n-1)}\right)^{-\frac{1}{2}} \mathrm{~d} a .
$$

For $n=3$ and $\Lambda>0$ (say), we have

$$
\begin{aligned}
I_{3} & =\int a\left(-a^{2}+\frac{\Lambda}{3} a^{4}+\frac{8 \pi G_{3} C_{1}}{3}\right)^{-\frac{1}{2}} \mathrm{~d} a \\
\text { (setting } \left.u=a^{2}\right) & =\frac{1}{2} \sqrt{\frac{3}{\Lambda}} \int\left(u^{2}-\frac{3}{\Lambda} u+\frac{8 \pi G_{3} C_{1}}{\Lambda}\right)^{-\frac{1}{2}} \mathrm{~d} u,
\end{aligned}
$$

whose integration is studied in [24] using the Weierstrass elliptic functions. Here we attempt a direct integration in view of Chebyshev's theorem and in terms of elementary functions. For this purpose, we assume

$$
32 \pi G_{3} C_{1} \Lambda<9
$$

(the critical case when $32 \pi G_{3} C_{1}=9$ is trivial) which allows us to rewrite (5.47) as

$$
I_{3}=\frac{1}{2} \sqrt{\frac{3}{\Lambda}} \int\left(\left(u-u_{1}\right)\left(u-u_{2}\right)\right)^{-\frac{1}{2}} \mathrm{~d} u,
$$

where

$$
u_{1,2}=\frac{3}{2 \Lambda} \pm \frac{1}{2} \sqrt{\left(\frac{3}{\Lambda}\right)^{2}-\frac{32 \pi G_{3} C_{1}}{\Lambda}}
$$

are ensured to be real and $u_{1}>u_{2}$ in view of (5.48). Setting $v=u-u_{1}$ (say), we get

$$
I_{3}=\frac{1}{2} \sqrt{\frac{3}{\Lambda}} \int v^{-\frac{1}{2}}\left(v+\left(u_{1}-u_{2}\right)\right)^{-\frac{1}{2}} \mathrm{~d} v
$$

which is integrable by the Chebyshev theorem. Hence, carrying out the integration (5.51), we find

$$
\begin{aligned}
I_{3} & =\frac{1}{2} \sqrt{\frac{3}{\Lambda}} \ln \left(-\frac{1}{2}\left(u_{1}+u_{2}\right)+u+\sqrt{u^{2}-\left(u_{1}+u_{2}\right) u+u_{1} u_{2}}\right)+C \\
& =\frac{1}{2} \sqrt{\frac{3}{\Lambda}} \ln \left(-\frac{3}{2 \Lambda}+u+\sqrt{u^{2}-\frac{3}{\Lambda} u+\frac{8 \pi G_{3} C_{1}}{\Lambda}}\right)+C \\
& =\frac{1}{2} \sqrt{\frac{3}{\Lambda}} \ln \left(-\frac{3}{2 \Lambda}+a^{2}+\sqrt{a^{4}-\frac{3}{\Lambda} a^{2}+\frac{8 \pi G_{3} C_{1}}{\Lambda}}\right)+C,
\end{aligned}
$$

where $C$ is an integration constant. 
It is readily checked that the condition (5.48) does not permit a big bang solution with $a(0)=0$. In fact, to stay in the real regime, it suffices to impose the condition

$$
a(0) \geq \sqrt{u_{1}}=\left(\frac{3}{2 \Lambda}+\left[\left(\frac{3}{2 \Lambda}\right)^{2}-\frac{8 \pi G_{3} C_{1}}{\Lambda}\right]^{\frac{1}{2}}\right)^{\frac{1}{2}}
$$

for the initial value of the scale factor. Hence the expanding-universe solution following from (5.52) is given by the formula

$$
a^{2}+\sqrt{a^{4}-\frac{3}{\Lambda} a^{2}+\frac{8 \pi G_{3} C_{1}}{\Lambda}}=\frac{3}{2 \Lambda}+C_{0} \mathrm{e}^{2 \sqrt{\frac{\Lambda}{3}} t},
$$

where $C_{0}>0$ is a constant depending on $a(0)$. Consequently, we have the following exponential growth pattern for the asymptotic form of the solution:

$$
a(t) \sim \sqrt{\frac{C_{0}}{2}} \mathrm{e}^{\sqrt{\frac{\pi}{3}} t}, \quad t \rightarrow \infty,
$$

which spells out the role of the cosmological constant $\Lambda$ clearly.

On the other hand, it can be examined that $I_{n}$, when $n>3$, does not allow a reduction into an integration involving a binomial differential so that the Chebyshev theorem is applicable.

\subsection{Friedmann's Equation in a Chern-Simons Modified Gravity Theory}

Following Jackiw and Pi [25], the Chern-Simons modified gravity theory in four spacetime dimensions is based on the extended Hilbert-Einstein action

$$
S=\frac{1}{16 \pi G} \int\left\{\sqrt{-g} R+\frac{\theta}{4} * R R\right\} \mathrm{d}^{4} x
$$

where $R$ is the Ricci scalar, ${ }^{*} R R$ the Pontryagin term given by

$$
{ }^{*} R R={ }^{*} R_{\beta}^{\alpha \mu \nu} R_{\alpha \mu \nu}^{\beta},
$$

with $R_{\alpha \mu \nu}^{\beta}$ the Riemann tensor and ${ }^{*} R_{\beta}^{\alpha \mu \nu}=\frac{1}{2} \epsilon^{\mu \nu \mu^{\prime} \nu^{\prime}} R_{\beta \mu^{\prime} \nu^{\prime}}^{\alpha}$ the dual Riemann tensor, $\theta$ a prescribed quantity, and the cosmological constant is taken to be zero. Recall that the Pontryagin term ${ }^{*} R R$ may be represented as a total divergence [25, 26, 27]:

$$
\frac{1}{4} R R=\nabla_{\mu} K^{\mu}
$$

where the 4-current $K^{\mu}$ reads

$$
K^{\mu}=\epsilon^{\mu \nu \alpha \beta}\left(\Gamma_{\nu \mu^{\prime}}^{\nu^{\prime}} \partial_{\alpha} \Gamma_{\beta \nu^{\prime}}^{\mu^{\prime}}+\frac{2}{3} \Gamma_{\nu \mu^{\prime}}^{\nu^{\prime}} \Gamma_{\alpha \alpha^{\prime}}^{\mu^{\prime}} \Gamma_{\beta \nu^{\prime}}^{\alpha^{\prime}}\right),
$$


in terms of the Christoffel symbols, resembling a Chern-Simons invariant [28, 29].

In [30], a general Chern-Simons modified gravity model defined by the action

$$
S=\frac{1}{16 \pi G} \int\left\{\sqrt{-g} R+\frac{l}{4} \Theta^{*} R R-\frac{1}{2} \partial^{\mu} \Theta \partial_{\mu} \Theta\right\} \mathrm{d}^{4} x+S_{\text {mat }},
$$

is considered, where $\Theta$ is now a dynamical variable [31, of the flavor of the Bekenstein model [32, 33, 34, $l$ a coupling parameter, and $S_{\text {mat }}$ denotes an additional matter action contribution. In the Friedmann-Robertson-Walker metric limit governing a homogeneous and isotropic universe, the scalar field $\Theta$ is shown [30] to obey the dynamics

$$
\ddot{\Theta}+3 H \dot{\Theta}=0
$$

which gives rise to the first integral

$$
\dot{\Theta}=C a^{-3}
$$

where $C$ is a constant, and the energy conservation law reads

$$
\frac{\mathrm{d}}{\mathrm{d} t}\left(\rho+\frac{C^{2}}{2} a^{-6}\right)+3 H\left(\rho+P+C^{2} a^{-6}\right)=0
$$

which is seen to simplify into the usual one

$$
\dot{\rho}+3 H(\rho+P)=0,
$$

independent of the Chern-Simons dynamical constant $C$ since $H=\frac{\dot{a}}{a}$. On the other hand, the flat-space $(k=0)$ Friedmann equation is

$$
H^{2}=\frac{8 \pi G}{3}\left(\rho+\frac{C^{2}}{2} a^{-6}\right)
$$

which relies on $C$. Thus, with the linear equation of state

$$
P=(\gamma-1) \rho
$$

we read off from (5.64) the relation $\rho=\rho_{0} a^{-3 \gamma}$. Consequently we arrive at the modified Friedmann equation [30]:

$$
\left(\frac{\dot{a}}{a}\right)^{2}=\frac{8 \pi G}{3}\left(\rho_{0} a^{-3 \gamma}+\frac{C^{2}}{2} a^{-6}\right) \equiv \frac{8 \pi G}{3} \rho_{\mathrm{eff}},
$$

where $\rho_{\text {eff }}$ is the effective energy density given by

$$
\rho_{\mathrm{eff}}=\rho_{\mathrm{eff}}(a)=\rho_{0} a^{-3 \gamma}+\frac{C^{2}}{2} a^{-6}
$$

resembling another two-fluid model. 
Integrating (5.67), we find

$$
I=\int a^{2}\left(\rho_{0} a^{6-3 \gamma}+\frac{C^{2}}{2}\right)^{-\frac{1}{2}} \mathrm{~d} a= \pm 2 \sqrt{\frac{2 \pi G}{3}} t .
$$

In view of the Chebyshev theorem, we see that, when $\gamma$ is rational, the integral $I$ is elementary if and only if $\gamma$ assumes the values

$$
\begin{aligned}
& \gamma=2-\frac{1}{N}, \quad N= \pm 1, \pm 2, \ldots \\
& \gamma=2-\frac{2}{2 N+1}, \quad N=0, \pm 1, \pm 2, \ldots
\end{aligned}
$$

As some illustrations, we first choose $N$ among the sequence (5.70), say $N=1$ or $\gamma=1$ (dust), to get

$$
I=\frac{2}{3 \rho_{0}}\left(\rho_{0} a^{3}+\frac{C^{2}}{2}\right)^{\frac{1}{2}}+C_{1},
$$

where $C_{1}$ is an integration constant. Hence the big-bang initial condition $a(0)=0$ gives us the expanding solution

$$
a(t)=\left(6 \pi G \rho_{0} t^{2}+2 \sqrt{3 \pi G}|C| t\right)^{\frac{1}{3}}, \quad t \geq 0
$$

Similarly we choose $\gamma$ among the second sequence (5.71), say $N=1$ or $\gamma=\frac{4}{3}$ (radiation), to get

$$
I=\frac{a}{2 \rho_{0}}\left(\rho_{0} a^{2}+\frac{C^{2}}{2}\right)^{\frac{1}{2}}-\frac{C^{2}}{4 \rho_{0}^{\frac{3}{2}}} \ln \left(\sqrt{\rho_{0}} a+\left(\rho_{0} a^{2}+\frac{C^{2}}{2}\right)^{\frac{1}{2}}\right)+C_{1},
$$

where $C_{1}$ is an integration constant so that for the big-bang solution it is fixed to be

$$
C_{1}=\frac{C^{2}}{4 \rho_{0}^{\frac{3}{2}}} \ln \frac{|C|}{\sqrt{2}}
$$

From (5.74), we obtain the following asymptotic behavior for expanding solutions:

$$
a(t) \sim 2\left(\frac{2 \pi G \rho_{0}}{3}\right)^{\frac{1}{4}} t^{\frac{1}{2}}, \quad t \rightarrow \infty .
$$

So in both cases the solutions increase as power functions.

Thus, we have seen that, in the multiple fluid situations studied above, power-law growth, periodic oscillations, and exponential expansion may all occur. It is interesting to note that a very wide range of evolutionary behavior can be achieved in the context of appropriate single-fluid models [35] as well. See also [36]. 


\section{The Reduced Temperature Equation}

In this section we study the Friedmann equation in terms of the reduced temperature and conformal time. We will see that, in such a setting, although the Chebyshev theorem is no longer applicable, new insight regarding integrability may be acquired which complements what may be obtained in terms of the scale factor and cosmic time.

\subsection{Governing Equation in Conformal Time}

We follow [37] to consider the normalized Friedmann equation

$$
\frac{1}{a^{2}}\left(\frac{\mathrm{d} a}{\mathrm{~d} t}\right)^{2}=\frac{C_{r}}{a^{4}}+\frac{C_{m}}{a^{3}}-\frac{k}{a^{2}}+\frac{\Lambda}{3},
$$

in 3 dimensions, where $C_{r}$ and $C_{m}$ are radiation and matter parameters, respectively. In the zero cosmological constant limit, $\Lambda=0$, the equation can be integrated in view of the discussion in Section 5. In this section, we study what happens when we switch to the conformal time $\eta$ :

$$
\mathrm{d} t=a \mathrm{~d} \eta
$$

and reformulate the Friedmann equation in the reduced temperature

$$
\tau=\frac{1}{\sqrt{\Lambda_{c}} a}, \quad \sqrt{\Lambda_{c}}=\frac{2}{3 C_{m}},
$$

as in [37]. In terms of these new variables, the Friedmann equation (6.1) becomes [37]

$$
\left(\frac{\mathrm{d} \tau}{\mathrm{d} \eta}\right)^{2}=\alpha \tau^{4}+\frac{2}{3} \tau^{3}-k \tau^{2}+\frac{\lambda}{3},
$$

where

$$
\lambda=\frac{\Lambda}{\Lambda_{c}}, \quad \alpha=C_{r} \Lambda_{c}
$$

are dimensionless parameters called the reduced cosmological constant and the reduced radiation parameter. It is interesting to note [37] that the Hubble parameter now assumes the form

$$
H=\frac{\mathrm{d}}{\mathrm{d} t} \ln a=-\sqrt{\Lambda_{c}} \frac{\mathrm{d} \tau}{\mathrm{d} \eta}
$$

When $\alpha=0$, the integration of (6.4) may be carried out in terms of elliptic functions [37]. Besides, it is clear that, when $\lambda=0$ and $\alpha \neq 0, k \neq 0$, the question whether (6.4) can be integrated lies out of the reach of the Chebyshev theorem. Nevertheless, we show here that the latter case enjoys an exact integration as well as it is given in the cosmic time $t$ and the scale factor $a$. 


\subsection{Integration of Equation}

We will only consider the nontrivial case, $\alpha \neq 0$. Thus we obtain from (6.4) the integral $I= \pm \eta$ where, formally,

$$
\begin{aligned}
I & =\int \tau^{-1}\left(\alpha \tau^{2}+\frac{2}{3} \tau-k\right)^{-\frac{1}{2}} \mathrm{~d} \tau \\
& =\int \tau^{-1}\left(\alpha\left[\tau+\frac{1}{3 \alpha}\right]^{2}-\left[\frac{1}{9 \alpha}+k\right]\right)^{-\frac{1}{2}} \mathrm{~d} \tau,
\end{aligned}
$$

which spells out another trivial case, $9 \alpha k=-1$. So we will only consider the nontrivial case, $9 \alpha k \neq-1$, below. Positivity of the quantity under the square root in (6.7) requires $\alpha>0$ or

$$
\frac{1}{9 \alpha}+k<0
$$

This observation splits our discussion into a few separate cases.

(i) If $\frac{1}{9 \alpha}+k>0$, then necessarily we have $\alpha>0$ in view of (6.7). Hence $\frac{1}{9}+\alpha k>0$. Now set

$$
\tau_{1,2}=\frac{-\frac{1}{3} \pm \sqrt{\frac{1}{9}+\alpha k}}{\alpha} .
$$

Introduce the new variable $v$ :

$$
\left(\left(\tau-\tau_{1}\right)\left(\tau-\tau_{2}\right)\right)^{\frac{1}{2}}=v\left(\tau-\tau_{1}\right)
$$

Then $\tau=\left(\tau_{1} v^{2}-\tau_{2}\right) /\left(v^{2}-1\right)$ and $\left(\left(\tau-\tau_{1}\right)\left(\tau-\tau_{2}\right)\right)^{\frac{1}{2}}=-\left(\tau_{2}-\tau_{1}\right) v /\left(v^{2}-1\right)$. Then (6.7) becomes

$$
\begin{aligned}
\sqrt{\alpha} I & =\int \tau^{-1}\left(\left(\tau-\tau_{1}\right)\left(\tau-\tau_{2}\right)\right)^{-\frac{1}{2}} \mathrm{~d} \tau \\
& =-2 \int \frac{\mathrm{d} v}{\tau_{1} v^{2}-\tau_{2}} .
\end{aligned}
$$

If $k=-1$, we have $\tau_{2}<\tau_{1}<0$ and

$$
\tau_{1} \tau_{2}=\frac{1}{\alpha}, \quad \frac{\tau_{2}}{\tau_{1}}=\frac{1}{\alpha}\left(\frac{1}{3}+\sqrt{\frac{1}{9}-\alpha}\right)^{2} .
$$

Hence we obtain

$$
I=\ln \left|\frac{3 \sqrt{\alpha} v-(1+\sqrt{1-9 \alpha})}{3 \sqrt{\alpha} v+(1+\sqrt{1-9 \alpha})}\right|+C,
$$

where and in sequel $C$ denotes an integration constant. If $k=1$, then $\tau_{2}<0<\tau_{1}$ and

$$
\frac{\tau_{2}}{\tau_{1}}=-\frac{1}{\alpha}\left(\frac{1}{3}+\sqrt{\frac{1}{9}+\alpha}\right)^{2} .
$$


So we obtain

$$
I=-2 \arctan \frac{3 \sqrt{\alpha} v}{1+\sqrt{1+9 \alpha}}+C .
$$

(ii) If $\frac{1}{9 \alpha}+k<0$, then both subcases, $\alpha>0$ and $\alpha<0$, are allowed. Hence, for $\alpha>0$, we have $k=-1$. So, taking $\tau+\frac{1}{3 \alpha}=u, u=\sqrt{-\frac{1}{\alpha}\left(-1+\frac{1}{9 \alpha}\right)} \tan \theta$, and $\tan \frac{\theta}{2}=v$, consecutively, we obtain

$$
\begin{aligned}
I & =\frac{1}{\sqrt{\alpha}} \int \tau^{-1}\left(\left[\tau+\frac{1}{3 \alpha}\right]^{2}-\frac{1}{\alpha}\left[-1+\frac{1}{9 \alpha}\right]\right)^{-\frac{1}{2}} \mathrm{~d} \tau \\
& =6 \sqrt{\alpha} \int \frac{\mathrm{d} v}{v^{2}+6 \alpha \sqrt{-\frac{1}{\alpha}\left(-1+\frac{1}{9 \alpha}\right)} v-1} \\
& =\ln \left|\frac{v-v_{1}}{v-v_{2}}\right|+C
\end{aligned}
$$

where

$$
v_{1,2}=3 \sqrt{\alpha}\left(-\sqrt{1-\frac{1}{9 \alpha}} \pm 1\right)
$$

are the roots of the denominator of the integrand of the second integral in (6.16), which further renders (6.16) into a more explicit form

$$
I=\ln \left|\frac{v+\sqrt{9 \alpha-1}-3 \sqrt{\alpha}}{v+\sqrt{9 \alpha-1}+3 \sqrt{\alpha}}\right|+C .
$$

Similarly, for $\alpha<0$, both $k=-1$ and $k=1$ are allowed. So, taking $T+\frac{1}{3 \alpha}=u$, $u=\sqrt{\frac{1}{\alpha}\left(k+\frac{1}{9 \alpha}\right)} \sin \theta$, and $\tan \frac{\theta}{2}=v$, consecutively, we obtain

$$
I=6 \sqrt{-\alpha} \int \frac{\mathrm{d} v}{v^{2}-6 \alpha \sqrt{\frac{1}{\alpha}\left(k+\frac{1}{9 \alpha}\right)} v+1} .
$$

Note that the discriminant of the denominator of the integrand of (6.19) is

$$
\Delta=36 \alpha k \text {. }
$$

In the nontrivial situation, $k \neq 0$, the condition $\alpha<0$ leads to $\Delta>0$ when $k=-1$ and $\Delta<0$ when $k=1$. Thus, we arrive at

$$
I= \begin{cases}-\ln \left|\frac{v+\sqrt{1-9 \alpha}-3 \sqrt{-\alpha}}{v+\sqrt{1-9 \alpha}+3 \sqrt{-\alpha}}\right|+C, & k=-1, \\ 2 \arctan \frac{v+\sqrt{9 \alpha+1}}{3 \sqrt{-\alpha}}+C, & k=1 .\end{cases}
$$

It is interesting to notice the similarities between (6.13), (6.18), and the first formula in (6.21), and between (6.15) and the second formula in (6.21). 


\section{Friedmann Type Equations in Other Contexts}

It is worth mentioning that the Friedmann type equations are also encountered in numerous other physical situations out of the remit of relativistic cosmology. Below we list a few examples. By this means one may hope to extend the range of the applications of the Chebyshev theorem but more generally to gain insight into the underlying geometry behind the Friedmann equation and to spot useful ways of representing the solutions. One particular feature that emerges is that many of the solutions may conveniently be represented as roulettes. This will be the subject of a future paper. For the present we content ourselves with pointing out some correspondences.

We begin by noting that if $k=1$ we have encountered the Friedmann equation in three different but equivalent forms

$$
\begin{gathered}
\frac{1}{a^{2}}\left(\frac{\mathrm{d} a}{\mathrm{~d} t}\right)^{2}+\frac{1}{a^{2}}=\rho(a), \\
\left(\frac{\mathrm{d} a}{\mathrm{~d} \eta}\right)^{2}+a^{2}=a^{4} \rho(a), \\
\left(\frac{\mathrm{d} \tau}{\mathrm{d} \eta}\right)^{2}+\tau^{2}=\rho\left(\frac{1}{\tau}\right),
\end{gathered}
$$

where $\mathrm{d} \eta=a^{-1} \mathrm{~d} t$ and $\tau=\frac{1}{a}$.

Note in this section we will adopt units in which $\frac{16 \pi G_{n}}{n(n-1)}=1$.

\subsection{Refraction in a Horizontally Stratified Medium}

Snell's law for the trajectory of a light ray $y=y(x)$ in a horizontally stratified medium reads

$$
\left(\frac{y^{\prime}}{y}\right)^{2}+\frac{1}{y^{2}}=\frac{n^{2}}{K^{2} y^{2}},
$$

where $K>0$ is a constant and $n=n(y)$ is the refractive index. Equation (7.4) is clearly of the $k=1$ Friedmann equation form with $y(x)$ corresponding to the scale factor $a(t)$. A direct integration gives us

$$
I=\int\left(\frac{n^{2}(y)}{K^{2}}-1\right)^{-\frac{1}{2}} \mathrm{~d} y= \pm x .
$$

As an example, consider

$$
n(y)=A y^{\alpha},
$$

where $A, \alpha$ are constants, $A \neq 0$. This corresponds to a barotropic equation of state with $3 \gamma=2(1-\alpha)$.

Applying the Chebyshev theorem, we see that all the integrable cases for $\alpha$ are:

$$
\alpha=\frac{1}{N}, \quad N= \pm 1, \pm 2, \ldots
$$


In particular, dark energy, radiation and dust correspond to $\alpha=1,-1,-\frac{1}{2}$, respectively. Explicitly we have

$$
I= \begin{cases}-\frac{A^{2}}{K^{2}} \arctan \sqrt{\frac{A^{2}}{K^{2} y}-1}-y \sqrt{\frac{A^{2}}{K^{2} y}-1}+C, & \alpha=-\frac{1}{2}, \\ -\sqrt{\frac{A^{2}}{K^{2}}-y^{2}}+C, & \alpha=-1, \\ \frac{K}{A} \ln \left|\frac{A}{K} y+\sqrt{\left(\frac{A y}{K}\right)^{2}-1}\right|+C, & \alpha=1,\end{cases}
$$

where $C$ is an integration constant.

Note that if $\alpha>0$ the refractive index increases with height, the rays are bent upwards, and this gives rise to a typical mirage effect. For example in the case of $\alpha=1$, i.e. dark energy, the rays are catenary curves. By contrast if $\alpha<0$, the refractive index decreases with height and the rays are bent downwards. If $\alpha=-1$, i.e. for radiation, the rays are semi-circles and for $\alpha=-\frac{1}{2}$, i.e. for dust, the rays are cycloids. In fact all three curves are roulettes. The case of dust, $\alpha=-\frac{1}{2}$ is equivalent to the brachistochone problem.

Another interesting case is when the index $n$ in (7.4) assumes the form

$$
n(y)=\frac{A y}{1+y},
$$

where $A$ is a constant satisfying

$$
\sigma=\frac{A}{K}>1
$$

The refractive index increases upwards and the rays are bent upwards. Hence the integral $I$ in (7.5) becomes

$$
\begin{aligned}
I= & \int \frac{1+y}{\sqrt{\left(\sigma^{2}-1\right) y^{2}-2 y-1}} \mathrm{~d} y \\
= & \frac{1}{\sqrt{\sigma^{2}-1}} \int(1+y)\left(\left[y-\frac{1}{\sigma-1}\right]\left[y+\frac{1}{\sigma+1}\right]\right)^{-\frac{1}{2}} \mathrm{~d} y, \\
& y>\frac{1}{\sigma-1} \quad \text { or } \quad y<-\frac{1}{\sigma+1},
\end{aligned}
$$

which in view of the Chebyshev theorem may be integrated similarly as done in Section 6 and is omitted. Here, we simply write down the result:

$$
I=\frac{1}{\sigma^{2}-1} \sqrt{\left(\sigma^{2}-1\right) y^{2}-2 y-1}+\frac{\sigma^{2}}{\left(\sigma^{2}-1\right)^{\frac{3}{2}}} \ln \left|y-\frac{1}{\sigma^{2}-1}+\sqrt{y^{2}-\frac{2 y+1}{\sigma^{2}-1}}\right|+C,
$$

where $C$ is an integration constant.

A difficult case is when the index $n$ is given by

$$
n(y)=\frac{C_{0} y^{2}}{\left(1+y^{2}\right)^{2}}, \quad C_{0}>0 .
$$

This problem lies beyond the reach of the Chebyshev theorem. 


\subsection{Soap Films and Glaciated Valleys}

Soap films are modeled by surfaces which extremize surface area for fixed volume enclosed. This is equivalent to their being of constant mean curvature. Thus if the soap film is a surface of revolution obtained by revolving the curve $y=y(x)$ about the $x$-axis we have

$$
\delta \int\left(y \sqrt{1+\left(y^{\prime}\right)^{2}}-\lambda y^{2}\right) \mathrm{d} x=0
$$

where $\lambda$ is a Lagrange multiplier enforcing the constant volume constraint. The EulerLagrange equation is

$$
-\frac{y^{\prime \prime}}{\left(1+y^{\prime 2}\right)^{\frac{3}{2}}}+\frac{1}{\left(1+y^{\prime 2}\right)^{\frac{1}{2}}}=2 \lambda
$$

which is the statement that the surface has constant mean curvature since the principal curvatures are

$$
\frac{1}{R_{1}}=-\frac{y^{\prime \prime}}{\left(1+y^{\prime 2}\right)^{\frac{3}{2}}}, \quad \frac{1}{R_{2}}=\frac{1}{y\left(1+y^{\prime 2}\right)^{\frac{1}{2}}} .
$$

Noether's theorem gives a first integral

$$
-\frac{y}{\sqrt{1+y^{\prime 2}}}+\lambda y^{2}=\text { constant }=c
$$

which may be written in the Friedmann form

$$
\left(\frac{y^{\prime}}{y}\right)^{2}+\frac{1}{y^{2}}=\frac{1}{\left(c-\lambda y^{2}\right)^{2}} .
$$

One has the correspondence

$$
a \leftrightarrow y, \quad t \leftrightarrow x,, \quad \rho \leftrightarrow \frac{1}{\left(c-\lambda y^{2}\right)^{2}}
$$

Note that in $n$ spatial dimensions, when the $S O(2)$ invariance is replaced by the $S O(n-1)$ invariance, $\lambda y^{2}$ must be replaced by $y^{n-1}$ (see [43]). It was discovered by Delaunay [41, 42] that we get in this way the locus of the focus of a conic section rolling without slipping on a line. There are three cases, the parabolic catenary, the elliptic catenary, and the hyperbolic catenary. The surfaces of revolution are called the catenoid, the unduloid and the nodoid

If $n=3$ and $\lambda=0$ we have a minimal surface of revolution which is well known to be a catenary. This corresponds to dark energy. If $n=3$ and $\lambda \neq 0$ the solution may be given in terms of elliptic functions. If $n>3$ we require in general hypo-elliptic functions [43].

The case $c=0$ is rather pathological. Superficially, As a soap film it corresponds to a cylinder with $y^{\prime}=0$. This is a perfectly valid solution as a soap film. However its interpretation as a solution of the Friedmann equation it would correspond to a static closed universe supported by radiation with $p=\frac{1}{3} \rho>0$. (This is a well treated case in 
literature [44]. See also [1] for a systematic treatment.) However $\dot{a}=0$ does not satisfy the Raychaudhuri equation

$$
\frac{\ddot{a}}{a}=-\frac{4 \pi G}{3}(\rho+3 P)
$$

Alternatively if one sets $H=0$ in equations $(2,5)$ and $(2,6)$ one obtains a contradiction unless $\frac{1}{3} \rho+P=0$.

In fact it is easy to show that the solution of the $k=1$ Friedmann equation for radiation in terms of conformal time is given by

$$
a(\eta)=\frac{1}{\lambda} \cos \eta, \quad t=\frac{1}{\lambda} \sin \eta
$$

which corresponds to a semi-circle in $(x, y)$ space and hence a sphere when revolved around the $x$ axis.

The example above was based on a variational principle and may be further generalized. The Euler-Lagrange equations of the variational problem

$$
\delta \int L\left(y, y^{\prime}\right) \mathrm{d} x=0
$$

where

$$
L=f(y) \sqrt{1+\left(y^{\prime}\right)^{2}}-g(y)
$$

admit the first integral

$$
-\frac{f}{\sqrt{1+\left(y^{\prime}\right)^{2}}}+g=c
$$

whence

$$
\left(\frac{y^{\prime}}{y}\right)^{2}+\frac{1}{y^{2}}=\frac{f^{2}}{y^{2}(c-g)^{2}}
$$

An interesting example is provided by a theory of the shape of glaciated valleys [45] for which $f=y$ and $g=\lambda y$, where the constant $\lambda$ is a Lagrange multiplier. Thus we have

$$
\left(\frac{y^{\prime}}{y}\right)^{2}+\frac{1}{y^{2}}=\frac{1}{(\lambda y-c)^{2}} \text {. }
$$

That is,

$$
\left(y^{\prime}\right)^{2}=\frac{y^{2}}{(\lambda y-c)^{2}}-1
$$

First set $u=\lambda y-c$, assuming $\lambda \neq 0$ and $c \neq 0$, otherwise it is trivial. Then we have

$$
\left(u^{\prime}\right)^{2}=\frac{\left(1-\lambda^{2}\right) u^{2}+2 c u+c^{2}}{u^{2}} .
$$

Thus we may assume $\lambda \neq \pm 1$ otherwise it is trivial. With $\lambda \neq \pm 1$, we use the transformation

$$
w=\left(\lambda^{2}-1\right) \frac{y}{c}-\lambda
$$


for comparison with the result in [45] to recast the equation (7.27) into

$$
\left(w^{\prime}\right)^{2}=\frac{\left(\lambda^{2}-1\right)^{3}}{c^{2}} \frac{\left(1-w^{2}\right)}{(\lambda w+1)^{2}}
$$

which can be integrated in view of the Chebyshev theorem. Carrying out the integration, we have

$$
\pm \frac{\left(\lambda^{2}-1\right)^{\frac{3}{2}}}{|c|} x=-\lambda \sqrt{1-w^{2}}+\arcsin w+C, \quad w^{2}<1 \quad \text { if } \lambda^{2}>1
$$

as given in [45], and

$$
\pm \frac{\left(1-\lambda^{2}\right)^{\frac{3}{2}}}{|c|} x=\lambda \sqrt{w^{2}-1}+\ln \left|w+\sqrt{w^{2}-1}\right|+C, \quad w^{2}>1 \text { if } \lambda^{2}<1
$$

where $C$ is an integration constant.

The case $c=0$ deserves special treatment. As a Friedmann equation it corresponds to a barotropic equation of state with $\rho>0$ but $\rho+3 P=0$. It thus permits a solution for which $\dot{a}=0$. In general the solution is linear in conformal time. This is consistent with the equation of state just violates the strong energy condition.

\subsection{Catenary of Equal Strength}

This curve satisfies ([48] p. 305 see also [50] p. 192)

$$
\frac{y^{\prime \prime}}{\left(1+\left(y^{\prime}\right)^{2}\right)}=\frac{1}{c}
$$

with first integral

$$
\arctan y^{\prime}=\frac{x}{c}+A
$$

whence

$$
y=B-c \ln \left|\cos \left(\frac{x}{c}+A\right)\right| .
$$

Inverting (7.34) for $y^{\prime}$ to get $\left(y^{\prime}\right)^{2}$ as a function of $\cos ^{2}\left(\frac{x}{c}+A\right)$ and using (7.35) lead to

$$
\frac{1}{y^{2}}+\left(\frac{y^{\prime}}{y}\right)^{2}=\frac{1}{y^{2}} \exp \left(\frac{2(y-B)}{c}\right)
$$

which is of the Friedmann form under the correspondence $(x, y) \leftrightarrow(t, a)$. Viewed as a closed Friedmann model it is time symmetric and exhibits a "Big Rip" [49], i.e a blow up of the scale factor, at finite time in the future and the time reverse of a big rip at finite time in the past. However if one evaluates the pressure $P$ the resulting equation of state does not seem to be very simple nor to have a simple physical interpretation.

The equation (7.36) is obviously beyond the reach of the Chebyshev theorem. 
Identifying the "matter density" $\rho$ as

$$
\rho=\frac{1}{y^{2}} \mathrm{e}^{\frac{2 y}{c}}
$$

and applying the "energy conservation law"

$$
\rho^{\prime}+3(\rho+P) \frac{y^{\prime}}{y}=0
$$

we deduce the equation of state relating the "pressure" to "matter density" as follows

$$
P=-\frac{1}{3} \rho-\frac{2}{3 c} \rho y=-\frac{1}{3} \rho-\frac{2}{3 c} \rho f(\rho),
$$

where $f$ is the inverse function defined by (7.37) if it exists. This equation is complicated. As a by-product, we have $\rho+P=\frac{2}{3} \rho\left(1-\frac{y}{c}\right)=\frac{2}{3} \rho\left(1-\frac{f(\rho)}{c}\right)$ whose sign is undetermined. However $\rho+3 P=-\frac{2 \rho y}{c}$ and therefore if $y>0$ and $c<0$ we have $\rho+P \geq 0$ and $\rho+3 P \geq 0$ and both the dominant and the strong energy conditions hold. On the other hand, if $c>0$ then the strong energy condition is violated.

\subsection{The Elastica of Bernoulli and the Capillary Curve}

Both of these curves satisfy (see [50] p.275)

$$
\pm \frac{y^{\prime \prime}}{\left(1+\left(y^{\prime}\right)^{2}\right)^{\frac{3}{2}}}=\frac{y}{b^{2}},
$$

with the first integral

$$
\mp \frac{1}{\sqrt{1+\left(y^{\prime}\right)^{2}}}-\frac{y^{2}}{2 b^{2}}=c .
$$

Turned upside down they give the shape of the "Hydrostatic Arch".

Rearranging (7.41) we have

$$
\frac{1}{y^{2}}+\left(\frac{y^{\prime}}{y}\right)^{2}=\frac{1}{y^{2}} \frac{1}{\left(c+\frac{y^{2}}{2 b^{2}}\right)^{2}},
$$

which is of the Friedmann form under the correspondence $(x, y) \leftrightarrow(t, a)$. In general, the solution is given by an elliptic integral but if $c=0, \pm 1$, then there are some elementary solutions (see below).

The special case $c=0$ has a linear equation of state corresponding to stiff matter with positive energy density and $P=\rho 2$ The Friedmann equation in terms of conformal time becomes

$$
\left(\frac{\mathrm{d} a}{\mathrm{~d} \eta}\right)^{2}+4 a^{4}=16 b^{4},
$$

\footnotetext{
${ }^{2}$ The same curves arise in the case of the Mylar balloon [52]. If one interchanges $x$ and $y$ one obtains (17.41) and the Mylar balloon corresponds to setting our integration constant $c$ to zero. More generally, any equation of the form $\frac{y^{\prime}}{\sqrt{1+\left(y^{\prime}\right)^{2}}}=f(x)$ may be converted to the Friedmann equation form in this way.
} 
whence

$$
a=a(0) \sqrt{\cos (2 \eta)} .
$$

Cosmic time is then given by an elliptic integral.

If $c=-1$ and we take the upper sign in (7.41) we can find a complete solution for $y$ and $x$ in elementary terms. For a direct evaluation using the integrals see below. In order to better understand the global behavior of the solutions, we first recall the traditional approach (see e.g. [50]) and we will take contact with the discussion based on direct integration below. We define $\psi$ to be $y^{\prime}=\tan \psi$, so $\psi$ is the angle the curve makes with the $x$-axis. the first integral (7.41) then becomes

$$
c \mp \cos \psi=\frac{y^{2}}{2 b^{2}} .
$$

Setting $c=-1$ and taking the upper sign in (7.45) we have

$$
y=2 b \sqrt{\sin \left(\frac{\psi}{2}\right)},
$$

where we have chosen the positive sign for the square root and we shall ultimately be interested in $\psi$ ranging from 0 to $2 \pi$. Now

$$
\frac{\mathrm{d} x}{\mathrm{~d} \psi}=\frac{\mathrm{d} x}{\mathrm{~d} s} \frac{\mathrm{d} s}{\mathrm{~d} \psi}
$$

where $\mathrm{d} s=\sqrt{1+\left(y^{\prime}\right)^{2}} \mathrm{~d} x$ along the curve, and using the fact that the curvature of a curve is given by

$$
\frac{y^{\prime \prime}}{\left(1+\left(y^{\prime}\right)^{2}\right)^{\frac{3}{2}}}=\frac{\mathrm{d} \psi}{\mathrm{d} s}
$$

and (7.35) we find that

$$
\frac{\mathrm{d} x}{\mathrm{~d} \psi}= \pm \frac{1}{2} b \frac{\cos \psi}{\sin \frac{\psi}{2}}= \pm \frac{1}{2} b\left(\frac{1}{\sin \frac{\psi}{2}}-2 \sin \frac{\psi}{2}\right)
$$

Taking the upper sign in (7.49) we see that $x$ increases with increasing $\psi$ from $\psi=0$ to $\psi=\frac{\pi}{2}$, it then decreases with increasing $\psi$ until $\psi=\frac{3 \pi}{2}$ and thereafter increases with increasing $\psi$ until $\psi=2 \pi$. Integrating (7.49) we find cosmic time to be given by

$$
t=x=b \ln \left(\tan \frac{\psi}{4}\right)+2 b \cos \frac{\psi}{2} .
$$

We see that as $\psi$ runs from 0 to $2 \pi, x$ runs from $-\infty$ to $+\infty$, and the curve, which is symmetric about the $y$-axis has a node on the $y$-axis and a loop running from the node and back thereafter continue to infinity. Between $\psi=\frac{\phi}{2}$ and $\psi=\frac{3 \pi}{2}$ the function $y(\psi)$ is double valued. On the branch from $\psi=0$ to $\psi=\pi$ the function $y(\psi)$ is monotonic 
increasing from zero and on the branch from $\psi=\pi$ to $\psi=2 \pi$ it is monotonic decreasing to zero. The maximum value of $y$ is $2 b$. At the node $y=2 b \sin \frac{\psi_{n}}{2}$ where $\psi_{n}$ satisfies

$$
\ln \left(\tan \frac{\psi_{n}}{4}\right)+2 \cos \frac{\psi_{n}}{2}=0 .
$$

Considered as a closed Friedmann model conformal time is given by

$$
\mathrm{d} \eta=\frac{\mathrm{d} x}{y}=\frac{\mathrm{d} t}{a}=\frac{1}{4}\left(\frac{1}{\sin ^{2} \frac{\psi}{2}}-2\right) \mathrm{d} \psi .
$$

That is, with a choice of integration constant,

$$
\eta=-\frac{1}{2} \cot \left(\frac{\psi}{2}+\psi-\pi\right) .
$$

If we take the branch that runs from $t=-\infty$ to $t=0$ we obtain a cosmological model expanding from zero size in the infinite past and reaching a finite size in finite time at $t=0$ at the node. The question then is what happens next? The continuation past the singular point is not obvious. If joined to the lower branch, then Hubble's constant $H=\frac{\dot{a}}{a}$ would jump from a positive to an equal negative value at $t=0$. However the Einstein equations would be violated and the jump in Hubble's constant would correspond to a spacelike hypersurface on which some matter instantaneously appears from nowhere.

A better alternative would be to follow the loop. The question is then what happens when one reaches $\psi=\frac{\pi}{2}$ ? Hubble's constant is given by

$$
H=\frac{1}{y} \frac{\mathrm{d} y}{\mathrm{~d} \psi} \frac{\mathrm{d} \psi}{\mathrm{d} x}=\frac{1}{\cos \frac{\psi}{2}} \frac{\sin ^{2} \frac{\psi}{2}}{\left(1-2 \sin ^{2} \frac{\psi}{2}\right)},
$$

which diverges as $\psi \rightarrow \frac{\pi}{2}$. This seems to be an example of a sudden singularity [51].

From (7.42) we can read off the "matter density" to be

$$
\rho=\frac{1}{y^{2}\left(c+\frac{y^{2}}{2 b^{2}}\right)^{2}} .
$$

Hence with $H=\frac{y^{\prime}}{y}$ we have

$$
\rho=-2 H\left(1+\frac{y^{2}}{\left(b^{2} c+\frac{1}{2} y^{2}\right)}\right) \rho .
$$

Comparing this result with the conservation law (7.38), we obtain

$$
\frac{1}{3} \rho+P=\frac{2 \rho y^{2}}{3\left(b^{2} c+\frac{1}{2} y^{2}\right)},
$$


which is positive for any $c \geq 0$. This is the strong energy condition, which implies the dominant energy condition $\rho+P>0$. If $c<0$, the sign of $\rho+P$ is generally undetermined.

One might also consider the top portion of the loop. This gives a time-symmetric closed universe which starts with a big bang at finite size $y=\sqrt{2} b$, with an infinite Hubble constant and reaches a time of maximum expansion at a finite time thereafter after which it recollapses to a big crunch.

In both of the two cases just discussed, the singularity is clear from (7.42) since if $c=-1$ the energy density diverges as $y \rightarrow \sqrt{2} b$ as $\frac{1}{(y-\sqrt{2} b)^{2}}$. This is reminiscent of the behavior of an equation of state with $P=-\frac{1}{3} \rho$.

Similar possibilities are possible for solutions for which $c \neq-1$. In particular if $c$ is negative similar singularities can arise. If $c>0$ the behavior is more like the $c=0$ case.

In general, setting

$$
u=\frac{y^{2}}{2 b^{2}}+c
$$

in (7.42) leads formally to the integration

$$
\pm \frac{\sqrt{2}}{|b|} x=\int \frac{u \mathrm{~d} u}{\sqrt{(u-c)\left(1-u^{2}\right)}} \equiv I_{c}
$$

which lies beyond the reach of the Chebyshev theorem unless $c=0, \pm 1$. So it is worthwhile to boil down the range of $c$ in which the problem is relevant. For this purpose, we rearrange (7.42) to get

$$
\left(c+\frac{y^{2}}{2 b^{2}}\right)^{2}\left(y^{\prime}\right)^{2}=\left([1-c]-\frac{y^{2}}{2 b^{2}}\right)\left(1+c+\frac{y^{2}}{2 b^{2}}\right) .
$$

So we see that the value $c>1$ is prohibited and $c=1$ gives rise to the trivial solution, $y \equiv 0$. For the remaining nontrivial integrable case, $c=-1$, we have

$$
\begin{aligned}
I_{-1} & =\sqrt{2} \operatorname{arctanh} \sqrt{\frac{1-u}{2}}-2 \sqrt{1-u}+C \\
& =\sqrt{2} \operatorname{arctanh} \sqrt{1-\frac{y^{2}}{4 b^{2}}}-2 \sqrt{2} \sqrt{1-\frac{y^{2}}{4 b^{2}}}+C,
\end{aligned}
$$

where $C$ is an integration constant. To make contact with our previous discussion we set

$$
\sqrt{1-\frac{y^{2}}{4 b^{2}}}=\cos \frac{\psi}{2}
$$

and use the identity

$$
\operatorname{arctanh} \cos \frac{\psi}{2}=\ln \cot \frac{\psi}{2}
$$




\subsection{Central Orbits}

Consider a particle moving in a plane with polar coordinates $r, \theta$. If $v$ is the speed and $V(r)$ the potential energy per unit mass then by energy conservation,

$$
\frac{1}{2}\left(\dot{r}^{2}+r^{2} \dot{\theta}^{2}\right)+V(r)=\mathcal{E}
$$

where $\mathcal{E}$ is the energy per unit mass. Angular momentum conservation gives

$$
r^{2} \dot{\theta}=h
$$

where $h$ is the angular momentum per unit mass. Thus

$$
\frac{1}{r^{4}}\left(\frac{\mathrm{d} a}{\mathrm{~d} \theta}\right)^{2}+\frac{1}{r^{2}}=-\frac{2}{h^{2}}(V(r)-\mathcal{E}) .
$$

Now setting $u=\frac{1}{r}$ we obtain

$$
\left(\frac{\mathrm{d} u}{\mathrm{~d} \theta}\right)^{2}+u^{2}=-\frac{2}{h^{2}}\left(V\left(\frac{1}{u}\right)-\mathcal{E}\right) .
$$

We see that this equation is of the same form as the reduced temperature version of the Friedmann equation (6.4) with $k=1$, under the correspondence $(u, \theta) \leftrightarrow(\tau, \eta)$ and bearing in mind that while $\theta$ is a periodic coordinate period $2 \pi$, conformal time $\eta$ is not periodic.

Central orbit problems arise from considering geodesics in spherically symmetric metrics. In this connection it is perhaps of interest that the equation (6.4) for the reduced temperature is of the same form as that obtained for null geodesics in the ReissnerNordstrom metric (see equation (51) in [38]) except the sign of the cosmological term is negative for real values of the electric charge.

\subsection{Spherical Symmetric Lenses}

A closely related example to the previous one is provided by applying Fermat's principle to a lens whose refractive index $n(r)$ depends on the radial coordinate $r$. The motion may be assumed to be in a plane through the origin and we have

$$
n^{2}(r)\left(\dot{r}^{2}+r^{2} \dot{\theta}^{2}\right)=1
$$

and

$$
n^{2} r^{2} \dot{\theta}=h=n r \sin i,
$$

where $h$ is a constant and $i$ is the inclination between the ray and the radial direction.

Thus

$$
\left(\frac{\mathrm{d} r}{r^{2} \mathrm{~d} \theta}\right)^{2}+\frac{1}{r^{2}}=\frac{n^{2}}{h^{2}}
$$


and so if $u=\frac{1}{r}$ we have

$$
\left(\frac{\mathrm{d} u}{\mathrm{~d} \theta}\right)^{2}+u^{2}=\frac{n^{2}\left(\frac{1}{u}\right)}{h^{2}}
$$

Note that while the lens problem is defined in the plane and hence $\theta$ is periodic of period $2 \pi$, no such restriction is placed on $t$. Thus the cosmological problem is defined on the universal covering space of the punctured plane.

Some examples are as follows:

- Maxwell's fish-eye lens is given by

$$
n(r)=\frac{n_{0}}{1+r^{2}}
$$

and thus

$$
\rho=\frac{n_{0}^{2}}{h^{2}\left(1+a^{2}\right)^{2}} .
$$

Comparing with (7.18) and Delauny's construction of soap films, it would appear that this case corresponds to the locus of the focus of an ellipse rolled without slipping along a line (i.e $\lambda c<0$ ). Now the optical metric of Maxwell's fish eye lens is that of a round 3-sphere pulled back to $\mathbb{R}^{3}$ by stereographic projection and hence all rays are circles. If these circles enclose the origin and lie in a plane through the origin they presumably map to the elliptic catenaries on the covering space.

- The Eaton lens has

$$
n=\sqrt{\frac{2 a}{r}-1}, \quad 0<r \leq a ; \quad n=1, \quad r>a .
$$

Thus for $r<a$, the solutions of which are portions of ellipses

$$
\frac{l}{r}=1+e \cos \left(\phi-\phi_{0}\right)
$$

with focus at the center $r=0$, semi-latus rectum $l$, and eccentricity $e$, where

$$
l=\frac{h^{2}}{a}, \quad e=\sqrt{1-\frac{b^{2}}{a^{2}}} .
$$

The ends of the semi-major axes of an elllipse are situated at a radial distance

$$
\frac{l}{1-e^{2}}
$$

from the focus at $r=0$. In our case one finds that this distance is $a$-independent of the impact parameter $h$. Because the refractive index is continuous across the boundary, rays entering the lens initially continue in the same direction are thus initially at one end of the semi-minor axis and initially moving perpendicularly to the semi-minor axis of their elliptical trajectory within the lens. It follows that they exit from the other end of the semi-minor axis in the opposite direction from that which enter the lens. Thus the Eaton lens behaves like a retroreflector: Any ray entering the lens exits in the opposite direction. 
- As pointed out in [47] the Eaton lens is dual in the sense of Arnold-Bohlin to the Luneburg lens for which

$$
n=\sqrt{2-\frac{r^{2}}{a^{2}}}, \quad r \leq a ; \quad n=1, \quad r>a .
$$

\section{Conclusion}

In this paper we have discussed the explicit integration of Friedmann's equations for equations of state more complicated than that of a single component fluid of linear barotropic form. In particular we have made use of a theorem of Chebyshev which gives sufficient conditions for explicit integrability. However we also found examples, while which are outside the scope of Chebyshev's result, nevertheless allow explicit integration. As well as the traditional form of the Friedmann equation is in terms of cosmic time, we have treated the case when the independent variable is conformal time. Less traditionally we have also considered the inverse of the scale factor (the so-called reduced temperature) as a function of conformal time. We have seen through a thorough study of the Chaplygin fluid model that integrable cases may often provide sharp insight into nonintegrable cases as well. In particular, based on some integrable examples, we have derived a universal formula for the asymptotic exponential growth rate of the scale factor in cosmic time, regardless whether the Friedmann equation falls in its integrable regime, and the formula reveals the coupled roles played by the conventional linear matter and nonconventional nonlinear matter, with regard to the presence of dark matter. Finally we have shown that all three forms of the Friedmann equation occur in areas of physics not directly connected with cosmology. This suggests that methods of dealing with the equation developed in those areas may transfer to the cosmological setting. In particular in a forthcoming paper we will illustrate this point by expressing its solutions as roulettes.

The research of Chen was supported in part by Henan Basic Science and Frontier Technology Program Funds under Grant No. 142300410110. Yang was partially supported by National Natural Science Foundation of China under Grant No. 11471100.

\section{References}

[1] S. Chen, G. W. Gibbons, Y. Li, and Y. Yang, Friedmann's equations in all dimensions and Chebyshev's theorem, J. Cosm. Astropart. Phys. 12 (2014) 035.

[2] M. P. Tchebichef, L'intégration des différentielles irrationnelles, J. Math. Pures Appl. 18 (1853) 87-111.

[3] E. A. Marchisotto and G.-A. Zakeri, An invitation to integration in finite terms, College Math. J. 25 (1994) 295-308.

[4] P. H. Chavanis, Relativistic self-gravitating Bose-Einstein condensates and cold baryons with a stiff equation of state, arXiv:1412.0005 [gr-qc]. 
[5] P. H. Chavanis, Cosmology with a stiff matter era, arXiv:1412.0743 [gr-qc].

[6] S. Sonego and V. Talamini, Qualitative study of perfect-fluid Friedmann-LamaîtreRobertson-Walker models with a cosmological constant, Am. J. Phys. 80 (2012) 670-679.

[7] S. Carneiro and C. Pigozzo, Observational tests for non-adiabatic Chaplygin gas, J. Cosm. Astropart. Phys. 1410 (2014) 060.

[8] P. P. Avelino, K. Bolejko, and G. F. Lewis, Nonlinear Chaplygin gas cosmologies, Phys. Rev. D 89 (2014) 103004.

[9] A. Yu. Kamenshchik, U. Moschellai, and V Pasquier, An alternative to quintessence, Phys. Lett. B 511 (2001) 265-268.

[10] M. C. Bento, O. Bertolami, and A. A. Sen, Generalized Chaplygin gas, accelerated expansion and dark energy-matter unification, Phys. Rev. 66 (2002) 043507.

[11] A. Vikman, Can dark energy evolve to the phantom? Phys. Rev. D 71 (2005) 023515.

[12] B. McInnes, The phantom divide in string gas cosmology, Nucl. Phys. B 718 (2005) 55-82.

[13] L. P. Chimento, R. Lazkoz, R. Maartens, and I. Quiros, Crossing the phantom divide without phantom matter, J. Cosm. Astropart. Phys. 0609 (2006) 004.

[14] S. Nesseris and L. Perivolaropoulos, Crossing the phantom divide: Theoretical implications and observational status, J. Cosm. Astropart. Phys. 0701 (2007) 018.

[15] H. Garcia-Compean, G. Garcia-Jimenez, O. Obregon, and C. Ramirez, Crossing the phantom divide in an interacting generalized Chaplygin gas, J. Cosm. Astropart. Phys. 0807 (2008) 016.

[16] J. Hogan, Unseen universe: Welcome to the dark side, Nature 448 (2007) 240-245.

[17] R. Caldwell and M. Kamionkowski, Cosmology: Dark matter and dark energy, Nature 458 (2009) 587-589.

[18] G. Ellis, R. Maartens, and M. MacCallum, Relativistic Cosmology, Cambridge University Press, Cambridge, U. K., 2012.

[19] R. Caldwell, M. Kamionkowski, and N. Weinberg, Phantom energy and cosmic doomsday, Phys. Rev. Lett. 91 (2003) 071301.

[20] R. J. Nemiroff and B. Patla, Adventures in Friedmann cosmology: A detailed expansion of the cosmological Friedmann equations, Am. J. Phys. 76 (2008) 265-276.

[21] R. J. Nemiroff and B. Patla, An exposition on Friedmann cosmology with negative energy densities, arXiv:1402.4522.

[22] A. Aviles, A. Bastarrachea-Almodovar, L. Campuzano, and H. Quevedo, Extending the generalized Chaplygin gas model by using geometrothermodynamics, Phys. Rev. D 86 (2012) 063508.

[23] H. Quevedo, Geometrothermodynamics, J. Math. Phys. 48 (2007) 013506. 
[24] E. Abdalla and L. A. L. Correa-Borbonet, The elliptic solutions to the Friedmann equation and the Verlinde's maps, arXiv:hep-th/0212205.

[25] R. Jackiw and S.-Y. Pi, Chern-Simons modification of general relativity, Phys. Rev. D 68 (2003) 104012.

[26] D. Grumiller and N. Yunes, How do black holes spin in Chern-Simons modified gravity? Phys. Rev. D 77 (2008) 044015.

[27] C. Furtado, T. Mariz, J. R. Nascimento, A. Yu. Petrov, and A. F. Santos, The Gödel solution in the modified gravity, Phys. Rev. D 79 (2009) 124039.

[28] S. S. Chern and J. Simons, Some cohomology classes in principal fiber bundles and their application to Riemannian geometry, Proc. Nat. Acad. Sci. USA 68 (1971) 791-794.

[29] S. S. Chern and J. Simons, Characteristic forms and geometric invariants, Ann. Math. 99 (1974) 48-69.

[30] C. Furtado, J. R. Nascimento, A. Yu. Petrov, and A. F. Santos, Dynamical Chern-Simons modified gravity and Friedmann-Robertson-Walker metric, arXiv:1005.1911.

[31] T. L. Smith, A. L. Erickcek, R. R. Caldwell, and M. Kamionkowski, The effects of ChernSimons gravity on bodies orbiting the Earth, Phys. Rev. D 77 (2008) 024015.

[32] J. Magueijo, H. Sandvik, and T. W. B. Kibble, Nielsen-Olesen vortex in varying-alpha theories, Phys. Rev. D 64 (2001) 023521.

[33] J. Menezes, P. P. Avelino, and C. Santos, Cosmic strings in Bekenstein-type models, J. Cosm. Astropart. Phys. 0502 (2005) 003.

[34] P. P. Avelino, C. J. A. P. Martins, and J. C. R. E. Oliveira, Linearized Bekenstein varying alpha models, Phys. Rev. D 70 (2004) 083506.

[35] J. D. Barrow, Graduated inflationary universes, Phys. Lett. B 235 (1990) 40-43.

[36] J. D. Barrow, String-driven inflationary and deflationary cosmological models, Nucl. Phys. B 310 (1988) 743-763.

[37] R. Coquereaux, The history of the universe is an elliptic curve, arXiv:1411.2192 [gr-qc].

[38] G. W. Gibbons and M. Vyska, The application of Weierstrass elliptic functions to Schwarzschild null geodesics, Class. Quant. Grav. 29 (2012) 065016, arXiv:1110.6508 [grqc].

[39] V. I. Arnold, Huygens and Barrow, Newton and Hooke, Birkhauser, Basel, 1990.

[40] M. K. Bohlin, Note sur le probléme des deux corps et sur une intégration nouvelle dans le probléme des trois corps, Bull Astrophysique 28 (1911) 113-119.

[41] C. Delaunay, Sur la surface de révolution dont la courbure moyenne ets constant, J. Math Pure et Appl. Sér 1 (6) (1841) 309-320. With a note appended by M. Sturm.

[42] J. Eells, The surfaces of Delaunay, Math. Intelligencer 9 (1987) 53-57. 
[43] W.-Y. Hsiang and W.-C. Yu, A generalization of a theorem of Delaunay J. Diff. Geom 17 (1981) 161-177.

[44] H. Stephani, D. Kramer, M. Maccallum, C. Hoenselaers, and E. Herlt, Exact Solutions of Einstein's Field Equations, Cambridge U. Press, Cambridge, U. K., 2003.

[45] F. Morgan, A note on cross-profile morphology for glacial valleys, Earth Surf. Process. Landforms 30 (2005) 513-514.

[46] J. Eaton, On spherically symmetric lenses, IRE Trans. Antennas Propagation 4 (1952) $66-71$.

[47] J. H. Hannay and T. M. Haeusser, Retroreflection by refraction, J. Mod. Optics 40 (1993) 1437-1442.

[48] E. J. Routh, A Treatise on Analytical Statics, Volume I, Cambridge U. Press, Cambridge, U. K. 1909.

[49] R. R. Caldwell, A Phantom menace? Cosmological consequences of a dark energy component with super-negative equation of state, Phys. Lett. B 545 (2002) 23-29.

[50] H. Lamb, Statics, Cambridge U. Press, Cambridge, U. K., 1909.

[51] J. D. Barrow, Sudden future singularities, Class. Quant. Grav. 21 (2004) L79-L82, arXiv: gr-qc/0403084.

[52] W. H. Paulsen, What is the shape of a Mylar Balloon, Amer. Math. Monthly 101 (1994) 953-958. 